Theoretical and Computational Fluid Dynamics manuscript No.

(will be inserted by the editor)

Samuel N. Stechmann · Andrew J. Majda • Boualem

Khouider

\title{
Nonlinear Dynamics of Hydrostatic Internal Gravity Waves
}

Submitted: September 23, 2007 / Revised: March 26, 2008 / Received: date / Accepted: date

\begin{abstract}
Stratified hydrostatic fluids have linear internal gravity waves with different phase speeds and vertical profiles. Here a simplified set of partial differential equations (PDE) is derived to represent the nonlinear dynamics of waves with different vertical profiles. The equations are derived by projecting the full nonlinear equations onto the vertical modes of two gravity waves, and the resulting equations are thus referred to here as the two-mode shallow water equations (2MSWE). A key aspect of the nonlinearities of the 2MSWE is that they allow for interactions between a background wind shear and propagating waves. This is important in the tropical atmosphere where horizontally propagating gravity waves interact together with wind shear and have source terms due to convection. It is shown here that the 2MSWE have nonlinear internal bore solutions, and the behavior of the nonlinear waves is investigated for different background wind shears. When a background shear is included, there is an asymmetry between the east- and westward propagating waves. This could be an important effect for the large-scale organization of tropical convection, since the convection is often not isotropic but organized on large scales by waves. An idealized illustration of this asymmetry is given for a background shear from the westerly wind burst phase of the Madden-Julian oscillation; the potential for organized convection is increased to the west of the existing convection by the propagating nonlinear gravity waves, which agrees qualitatively with actual observations. The ideas here should be useful for other physical applications as well.

Moreover, the 2MSWE have several interesting mathematical properties: they are a system of nonconservative PDE with a conserved energy, they are conditionally hyperbolic, and they are neither
\end{abstract}

Samuel N. Stechmann

Courant Institute of Mathematical Sciences

New York University

251 Mercer Street

New York, NY 10012 USA

Tel.: 212-998-3302

Fax: 212-995-4121

E-mail: stechman@cims.nyu.edu

Andrew J. Majda

Courant Institute of Mathematical Sciences

New York University

251 Mercer Street

New York, NY 10012 USA

Boualem Khouider

Department of Mathematics and Statistics

University of Victoria

PO BOX 3045 STN CSC

Victoria, BC

Canada V8W 3P4 
genuinely nonlinear nor linearly degenerate over all of state space. Theory and numerics are developed to illustrate these features, and these features are important in designing the numerical scheme. A numerical method is designed with simplicity and minimal computational cost as the main design principles. Numerical tests demonstrate that no catastrophic effects are introduced when hyperbolicity is lost, and the scheme can represent propagating discontinuities without introducing spurious oscillations.

Keywords Internal gravity waves · internal bores · stratified fluids · tropical atmospheric dynamics · convectively generated gravity waves $\cdot$ nonconservative PDE $\cdot$ computational methods for nonconservative PDE

PACS 47.35.Bb $\cdot 47.11 .-\mathrm{j} \cdot 92.60 .-\mathrm{e}$

\section{Introduction}

The nonlinear dynamics of waves are important in many physical applications involving interactions across different space and time scales. Energy can be transported upscale or downscale as in turbulence and wave-mean interaction theory; the properties of waves can change depending on the background state through which they propagate; and some phenomena are inherently multiscale and involve a combination of these effects [1-4].

The tropical atmosphere abounds with examples of nonlinear and multiscale effects of waves on many different scales, including the quasi-biennial oscillation [5], the Madden-Julian oscillation [6], convectively coupled equatorial waves $[7,8]$, squall lines and other mesoscale convective systems [9], and density currents and gravity waves generated by convective clouds [10-12]. For most of these examples, hydrostatic balance is a reasonable approximation because the aspect ratio (vertical length scale over horizontal length scale) is small. This corresponds to horizontal spatial scales of more than $100 \mathrm{~km}$ and time scales of more than 1 hour. On these scales, waves interact nonlinearly with background wind shear and with source terms due to convection. In a growing body of literature, these effects are shown to be mostly captured by two vertical Fourier modes [13-23]. While the important vertical modes have been identified, and while they have been used in many linear studies, the nonlinear interactions among the vertical modes have been largely ignored in simple models.

Here a set of simplified partial differential equations (PDE) is derived and analyzed for the nonlinear interactions between different vertical modes. The derivation is carried out by projecting the full equations of motion, the nonlinear hydrostatic Boussinesq equations, onto the vertical modes of two gravity waves. The projected equations are thus referred to here as the two-mode shallow water equations (2MSWE). This derivation is carried out in section 2.

The 2MSWE have several interesting mathematical properties: they are a system of nonconservative PDE with a conserved energy, they are conditionally hyperbolic, and they are neither genuinely nonlinear nor linearly degenerate over all of state space. Theory and numerics are developed to illustrate these features in sections $3-5$, and these features will be important in designing the numerical scheme.

A simple numerical method for the $2 \mathrm{MSWE}$ is presented in section 3 . The scheme is designed with simplicity and minimal computational cost as the main design principles. The idea is to split the equations into conservative and nonconservative parts and to solve each part with an appropriate method. The numerical scheme is tested with emphasis on conditional hyperbolicity (section 4) and breaking waves that resemble internal bores (a.k.a. density currents or gravity currents) [24-27] (section 5).

An important aspect of the nonlinearities of the 2MSWE is that they allow for the effects of a background wind shear interacting with propagating waves. This is emphasized in sections 5 and 6 . In section 6, it is shown that a background wind shear causes asymmetries between the westward- and eastward-propagating waves. This could have important implications for the large-scale organization of tropical convection, since waves propagating away from clouds can suppress or favor the formation of new convective clouds $[10,11]$. Furthermore, the background wind shear is also important for convection because it determines whether convection will be unorganized and scattered or whether it will become organized into a squall line or mesoscale convective system [28,29]. In other work, in order to represent these important effects of background wind shear, the authors are currently adding the nonlinear 
effects of the 2MSWE to a simplified model for organized tropical convection [18-23]. That model, which is called the multicloud model, includes the effects of water vapor and clouds through additional equations and nonlinear interactive source terms. The 2MSWE can be used in the multicloud model as a nonlinear dynamical core, since its dynamical core was originally linear for simplicity. Results with the multicloud model with the effect of background shear will be reported elsewhere in the near future.

\section{Derivation of the Two-Mode Shallow Water Equations (2MSWE)}

The setup considered here is a hydrostatic Boussinesq fluid with a background stratification that is linear with height. The fluid is bounded above and below by rigid lids where free slip boundary conditions are assumed: $\left.w\right|_{z=0, H}=0$. In this situation there is an infinite set of linear gravity waves with phase speeds $c_{j}$ and vertical profiles $\sin (j z)$ for $j=1,2,3, \cdots[1]$. The waves $c_{j}$ are decoupled from all waves $c_{k}(k \neq j)$ for the case of linear dynamics, but the waves become coupled in the case of nonlinear dynamics. In this section nonlinear effects are included to describe the interactions between the waves $c_{j}$ and $c_{k}(k \neq j)$. Here $c_{1}$ and $c_{2}$ are chosen because of their relevance to applications in atmospheric science [13-23]. The PDE for these interactions are derived by projecting the full nonlinear equations onto the dynamics for only the two waves $c_{1}$ and $c_{2}$.

Hydrostatic Boussinesq equations. Consider the equations for a rotating hydrostatic Boussinesq fluid $[1-3]$ :

$$
\begin{aligned}
\frac{D \mathbf{U}}{D t}+f(y) \mathbf{U}^{\perp} & =-\nabla P \\
\nabla \cdot \mathbf{U}+\frac{\partial W}{\partial z} & =0 \\
\frac{\partial P}{\partial z} & =g \frac{\Theta}{\theta_{r e f}} \\
\frac{D \Theta}{D t}+W \frac{d \theta_{b g}}{d z} & =0,
\end{aligned}
$$

where the material derivative is

$$
\frac{D}{D t}=\frac{\partial}{\partial t}+\mathbf{U} \cdot \nabla+W \frac{\partial}{\partial z}
$$

Here the horizonal velocity is $\mathbf{U}=(U(x, y, z, t), V(x, y, z, t))$, and $\nabla$ is the horizonal gradient operator: $\mathbf{U} \cdot \nabla=U \partial_{x}+V \partial_{y}$. The model parameters and scales are given in Table 1 . With the background potential temperature $\theta_{b g}(z)$ and the reference potential temperature $\theta_{\text {ref }}=300 \mathrm{~K}$, the total potential temperature of this model is

$$
\theta_{\text {total }}=\theta_{\text {ref }}+\theta_{b g}(z)+\Theta(x, y, z, t) .
$$

The pressure $P$ has been scaled by a reference density so its units are not the typical pressure units. The term $f(y) \mathbf{U}^{\perp}$ represents the Coriolis force, where $\mathbf{U}^{\perp}=(-V, U)$. In what follows, the equatorial $\beta$-plane approximation will be used $[1,2]$. This approximation replaces $f(y)$ with the first two terms of its Taylor expansion at the equator $(y=0)$. Since $f(y=0)=0$, this takes the form $f(y) \approx \beta y$, where the value of $\beta$ is used here to define reference length and time scales as shown in Table 1. Although this special form of $f(y)$ is used here, the ideas in this paper apply for a general Coriolis parameter $f(y)$ as well.

Using the scales defined in Table 1, (1) are nondimensionalized to give

$$
\begin{aligned}
\frac{D \mathbf{U}}{D t}+y \mathbf{U}^{\perp} & =-\nabla P \\
\nabla \cdot \mathbf{U}+\frac{\partial W}{\partial z} & =0 \\
\frac{\partial P}{\partial z} & =\Theta \\
\frac{D \Theta}{D t}+W & =0 .
\end{aligned}
$$


Table 1 Model parameters and scales.

\begin{tabular}{llll}
\hline Parameter & Derivation & Value & Description \\
\hline$\beta$ & & $2.3 \times 10^{-11} \mathrm{~m}^{-1} \mathrm{~s}^{-1}$ & Variation of Coriolis parameter with latitude \\
$\theta_{\text {ref }}$ & $300 \mathrm{~K}$ & Reference potential temperature \\
$g$ & & $9.8 \mathrm{~m} / \mathrm{s}^{2}$ & Gravitational acceleration \\
$H$ & & $16 \mathrm{~km}$ & Tropopause height \\
$N^{2}$ & $\left(g / \theta_{r e f}\right) d \theta_{b g} / d z$ & $10^{-4} \mathrm{~s}^{-2}$ & Buoyancy frequency squared \\
$c$ & $N H / \pi$ & $50 \mathrm{~m} / \mathrm{s}$ & Velocity scale \\
$L$ & $\sqrt{c / \beta}$ & $1500 \mathrm{~km}$ & Equatorial length scale \\
$T$ & $L / c$ & $8 \mathrm{hrs}$ & Equatorial time scale \\
$\bar{\alpha}$ & $H N^{2} \theta_{r e f} /(\pi g)$ & $15 \mathrm{~K}$ & Potential temperature scale \\
& $H / \pi$ & $5 \mathrm{~km}$ & Vertical length scale \\
& $H /(\pi T)$ & $0.2 \mathrm{~m} / \mathrm{s}^{2}$ & Vertical velocity scale \\
& $c^{2}$ & $2500 \mathrm{~m}^{2} \mathrm{~s}^{-2}$ & Pressure scale \\
\hline
\end{tabular}

The fluid is bounded above and below by rigid lids at the earth's surface $(z=0)$ and the top of the troposphere $(z=H=16 \mathrm{~km})$. The boundary condition at the surface is $W=0$, and the boundary condition at $z=H$ is also $W=0$. While the atmosphere has no such rigid lid at $z=H$, several studies have shown that this is a reasonable approximation $[10,15,18,19,30]$.

Vertical basis functions. There is a natural set of vertical basis functions for (2). The basis functions can be obtained by linearizing the equations, using separation of variables for the $z$-dependence, and solving the resulting Stürm-Liouville problem [1]. Besides their relevance as vertical eigenfuctions for the equations of motion, several of the basis functions also appear prominently in the tropical atmosphere due to cloud types associated with certain vertical modes [13-23]. These basis functions take the form of sinusoids:

$$
\begin{aligned}
& C_{0}(z)=1 \\
& C_{j}(z)=\sqrt{2} \cos (j z), \quad S_{j}(z)=\sqrt{2} \sin (j z), \quad j=1,2,3, \cdots
\end{aligned}
$$

where the upper lid is located at $z=\pi$ in nondimensional units. The inner product is then defined as

$$
\langle F(z), G(z)\rangle=\frac{1}{\pi} \int_{0}^{\pi} F(z) G(z) d z
$$

so that the $S$ 's and the $C$ 's are orthonormal bases: $\left\langle S_{i}, S_{j}\right\rangle=\delta_{i j}$ and $\left\langle C_{i}, C_{j}\right\rangle=\delta_{i j}$. The model variables are expanded as

$$
\begin{aligned}
\mathbf{U}(x, y, z, t)=\sum_{j=0}^{\infty} \mathbf{u}_{j}(x, y, t) C_{j}(z) & \Theta(x, y, z, t)=\sum_{j=1}^{\infty} \theta_{j}(x, y, t) j S_{j}(z) \\
P(x, y, z, t)=\sum_{j=0}^{\infty} p_{j}(x, y, t) C_{j}(z) & W(x, y, z, t)=\sum_{j=1}^{\infty} w_{j}(x, y, t) S_{j}(z) .
\end{aligned}
$$

Note that the convention here is to expand $\Theta$ in the basis $j S_{j}(z)$, not $S_{j}(z)$. This vertical structure is illustrated for the first few modes in Figure 1.

Vertical projection of linearized equations. Before dealing with the nonlinear equations (2), the linearized version is considered to illustrate the vertical projection in a simpler setting. A trivial background state $U=W=P=\Theta=0$ is used for the linearization. If the expansion (5) is then inserted into the linearized version of (2), and if the equations are projected onto each mode $j=0,1,2, \cdots$ using the inner product (4), then the variables for each mode $j$ solve a set of equations that is decoupled 
(a)

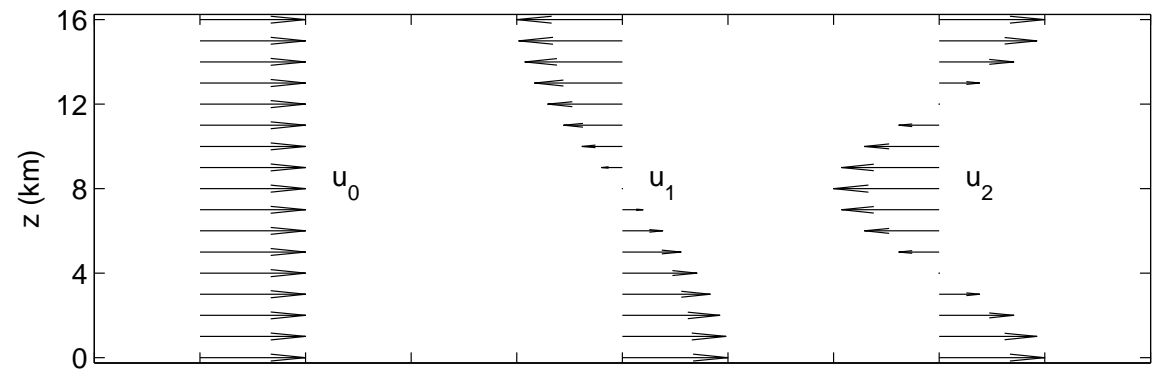

(b)

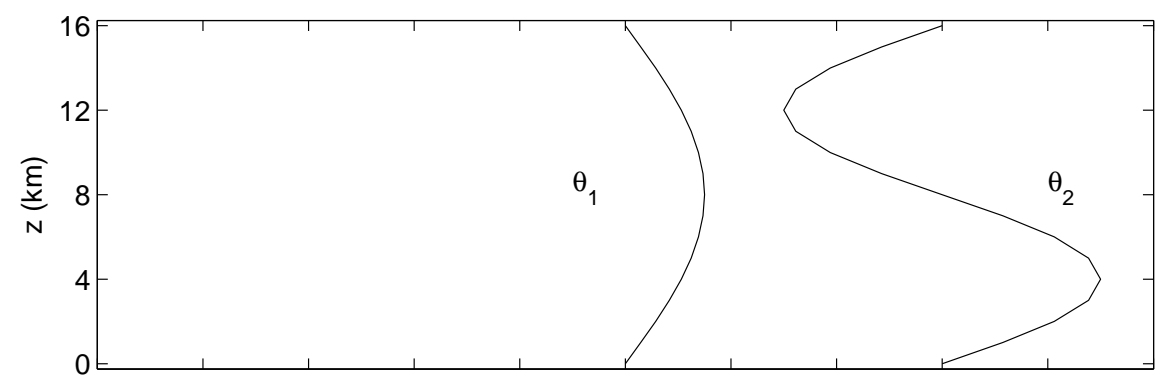

Fig. 1 Vertical profiles for the first few modes of velocity (a) and potential temperature (b), as described in (3) and (5).

from the other modes. For $j=0$ (the barotropic mode), the equations that arise are the rotating linear $2 \mathrm{D}$ incompressible fluid equations:

$$
\begin{aligned}
\frac{\partial \mathbf{u}_{0}}{\partial t}+y \mathbf{u}_{0}^{\perp}+\nabla p_{0} & =0 \\
\nabla \cdot \mathbf{u}_{0} & =0
\end{aligned}
$$

For each other mode $j=1,2,3, \cdots$ (the baroclinic modes), the equations that arise are the rotating linear shallow water equations:

$$
\begin{aligned}
\frac{\partial \mathbf{u}_{j}}{\partial t}+y \mathbf{u}_{j}^{\perp}-\nabla \theta_{j} & =0 \\
\frac{\partial \theta_{j}}{\partial t}-\frac{1}{j^{2}} \nabla \cdot \mathbf{u}_{j} & =0, \quad j=1,2,3, \cdots
\end{aligned}
$$

where the wavespeed for the $j$ th set of equations is $c_{j}=1 / j$ in nondimensional units. The hydrostatic and continuity equations of (2) give the relations

$$
\theta_{j}=-p_{j}, \quad w_{j}=-\frac{1}{j} \nabla \cdot \mathbf{u}_{j}, \quad j=1,2,3, \cdots
$$

The equations (6) and (7) have a rich variety of linear waves (see [1] for more details). While the equations have been written down here for an equatorial $\beta$-plane, the projection can also be carried out for a general Coriolis parameter $f(y)$.

Vertical projection of nonlinear equations. Now we return to the nonlinear hydrostatic Boussinesq equations in (2). Observations and simulations show that the 1st and 2nd baroclinic modes are the 
most important for many phenomena in the tropical atmosphere [13-23]. Therefore, the model variables are assumed to have the truncated form

$$
\begin{array}{ll}
\mathbf{U}=\mathbf{u}_{1} C_{1}+\mathbf{u}_{2} C_{2} & \Theta=\theta_{1} S_{1}+\theta_{2} 2 S_{2} \\
W=-\nabla \cdot \mathbf{u}_{1} S_{1}-\frac{1}{2} \nabla \cdot \mathbf{u}_{2} S_{2} & P=-\theta_{1} C_{1}-\theta_{2} C_{2} .
\end{array}
$$

When (2) are projected onto the 1st and 2 nd baroclinic modes, the result is the rotating two-mode shallow water equations (2MSWE):

$$
\begin{aligned}
& \left\{\begin{aligned}
\frac{\partial \mathbf{u}_{1}}{\partial t}+y \mathbf{u}_{1}^{\perp}-\nabla \theta_{1} & =-\frac{1}{\sqrt{2}}\left[\mathbf{u}_{1} \cdot \nabla \mathbf{u}_{2}+\mathbf{u}_{2} \cdot \nabla \mathbf{u}_{1}+2 \mathbf{u}_{2} \nabla \cdot \mathbf{u}_{1}+\frac{1}{2} \mathbf{u}_{1} \nabla \cdot \mathbf{u}_{2}\right] \\
\frac{\partial \theta_{1}}{\partial t}-\nabla \cdot \mathbf{u}_{1} & =-\frac{1}{\sqrt{2}}\left[2 \mathbf{u}_{1} \cdot \nabla \theta_{2}-\mathbf{u}_{2} \cdot \nabla \theta_{1}+4 \theta_{2} \nabla \cdot \mathbf{u}_{1}-\frac{1}{2} \theta_{1} \nabla \cdot \mathbf{u}_{2}\right]
\end{aligned}\right. \\
& \left\{\begin{aligned}
\frac{\partial \mathbf{u}_{2}}{\partial t}+y \mathbf{u}_{2}^{\perp}-\nabla \theta_{2} & =-\frac{1}{\sqrt{2}}\left[\mathbf{u}_{1} \cdot \nabla \mathbf{u}_{1}-\mathbf{u}_{1} \nabla \cdot \mathbf{u}_{1}\right] \\
\frac{\partial \theta_{2}}{\partial t}-\frac{1}{4} \nabla \cdot \mathbf{u}_{2} & =-\frac{1}{2 \sqrt{2}}\left[\mathbf{u}_{1} \cdot \nabla \theta_{1}-\theta_{1} \nabla \cdot \mathbf{u}_{1}\right]
\end{aligned}\right.
\end{aligned}
$$

Energy Conservation Define the energy density

$$
\mathcal{E}=\frac{1}{2}\left(\left|\mathbf{u}_{1}\right|^{2}+\left|\mathbf{u}_{2}\right|^{2}+\theta_{1}^{2}+4 \theta_{2}^{2}\right) .
$$

One can show that this is a conserved energy for smooth solutions of (10):

$$
\frac{\partial \mathcal{E}}{\partial t}+\nabla \cdot \mathcal{F}=0, \quad \mathcal{F}=-\theta_{1} \mathbf{u}_{1}-\theta_{2} \mathbf{u}_{2}+\frac{1}{\sqrt{2}}\left[\mathbf{u}_{1} \cdot \mathbf{u}_{2}\right] \mathbf{u}_{1}+\frac{1}{2 \sqrt{2}}\left|\mathbf{u}_{1}\right|^{2} \mathbf{u}_{2}+\sqrt{2} \theta_{1} \theta_{2} \mathbf{u}_{1}-\frac{1}{2 \sqrt{2}} \theta_{1}^{2} \mathbf{u}_{2}
$$

The form of this flux illustrates that the nonlinear terms in (10) can lead to energy exchanges between modes 1 and 2 .

Two-Mode SWE above the Equator. The main focus of this paper is on the nonlinear wave interactions between different vertical modes. To illustrate the physical and numerical issues in the simplest possible setting, the 2MSWE will be studied for the rest of this paper in a 1D setting above the equator $(y=0)$. This will eliminate the dispersive equatorial waves and leave only $1 \mathrm{D}$ non-dispersive gravity waves. In this setup above the equator, the Coriolis parameter $f(y)$ vanishes, and the north-south $y$-depenedence of the flow and the north-south velocities $v_{j}$ are set to zero. With these simplifications, the $2 \mathrm{MSWE}$ (10) become

$$
\begin{aligned}
& \left\{\begin{array}{l}
\frac{\partial u_{1}}{\partial t}-\frac{\partial \theta_{1}}{\partial x}=-\frac{3}{\sqrt{2}}\left[u_{2} \frac{\partial u_{1}}{\partial x}+\frac{1}{2} u_{1} \frac{\partial u_{2}}{\partial x}\right] \\
\frac{\partial \theta_{1}}{\partial t}-\frac{\partial u_{1}}{\partial x}=-\frac{1}{\sqrt{2}}\left[2 u_{1} \frac{\partial \theta_{2}}{\partial x}-u_{2} \frac{\partial \theta_{1}}{\partial x}+4 \theta_{2} \frac{\partial u_{1}}{\partial x}-\frac{1}{2} \theta_{1} \frac{\partial u_{2}}{\partial x}\right]
\end{array}\right. \\
& \left\{\begin{array}{c}
\frac{\partial u_{2}}{\partial t}-\frac{\partial \theta_{2}}{\partial x}=0 \\
\frac{\partial \theta_{2}}{\partial t}-\frac{1}{4} \frac{\partial u_{2}}{\partial x}=-\frac{1}{2 \sqrt{2}}\left[u_{1} \frac{\partial \theta_{1}}{\partial x}-\theta_{1} \frac{\partial u_{1}}{\partial x}\right]
\end{array}\right.
\end{aligned}
$$

These will be the equations used in the rest of this paper. This system is more mathematically tractable than the $2 \mathrm{D}$ version in $(10)$, and, as will be shown in subsequent sections, it still allows for interesting interactions between the different vertical modes. 
Note that, in this 1D setting, the barotropic mode must be a constant due to the incompressibility constraint: $0=\operatorname{div} \mathbf{u}_{0}=\partial u_{0} / \partial x$. In $2 \mathrm{D}$, however, the barotropic mode is active. The $2 \mathrm{D}$ case of interactions between the barotropic mode and the 1st baroclinic mode was studied in [31], and a numerical scheme for those equations was designed in $[32,33]$.

Comparison with two-layer shallow water equations. Traditionally, the simple models used for studying stratification, shear, and internal waves in the atmosphere and ocean are the multi-layer shallow water equations (SWE) [2,34]. The two-layer SWE and the 2MSWE (13) have several important differences and similarities, both physical and mathematical. In terms of their physical properties, they are quite different. The two-layer SWE describe flows with two layers of constant densities $\left(\rho_{1}\right.$ and $\left.\rho_{2}\right)$, and the dynamical variables are the velocity in each layer $\left(u_{1}(x, t)\right.$ and $\left.u_{2}(x, t)\right)$ and the thickness of each layer $\left(h_{1}(x, t)\right.$ and $\left.h_{2}(x, t)\right)$. Since the density has no horizontal variations within each layer, the model does not represent thermodynamic processes (see [35] for a discussion of layer models that include thermodynamic effects). In contrast, the 2MSWE include thermodynamic effects through the potential temperature $\left(\theta_{1}(x, t)\right.$ and $\left.\theta_{2}(x, t)\right)$. In addition, the two-layer SWE include a free upper surface, and the vertical structure of the flow consists of the barotropic mode and the 1st baroclinic mode. In contrast, the 2MSWE have a rigid upper lid, and the vertical structure of the flow consists of the 1st and 2nd baroclinic modes (the barotropic mode is inactive in 1D in the presence of a rigid upper lid).

In terms of their mathematical form, the 2MSWE and the two-layer SWE have several similarities: both are systems of nonconservative PDE in four variables, both have a conserved energy, both are conditionally hyperbolic, and both have eigenstructures that are analytically intractable. One difference is in the behavior of nonlinear waves. The baroclinic mode of the two-layer SWE is like all of the modes of the 2MSWE in that it is neither genuinely nonlinear nor linearly degenerate over all of phase space. The barotropic mode of the two-layer SWE, however, is genuinely nonlinear.

There are also models for compressible two-phase flow that share many of these mathematical properties. See, for instance, $[36,37]$ and references therein.

\section{Numerical Methods}

In this section, a numerical method for the 2MSWE is proposed, and a convergence test is carried out to verify second-order convergence. The 2MSWE (13) have the following properties that must be considered when designing a numerical scheme:

- They are nonconservative, i.e., they take the form

$$
\frac{\partial \mathbf{u}}{\partial t}+A(\mathbf{u}) \frac{\partial \mathbf{u}}{\partial x}=0
$$

where $\mathbf{u}=\left(u_{1}, \theta_{1}, u_{2}, \theta_{2}\right)^{T}$ and $A(\mathbf{u})$ is a matrix that cannot be written as $\partial \mathbf{F} / \partial \mathbf{u}$ for any flux $\mathbf{F}$.

- The energy in (11) plays the role of a convex entropy function, and an entropy/entropy-flux pair $(\Phi, \Psi)$ is formed by identifying $\Phi=\mathcal{E}$ and $\Psi=\mathcal{F}$ from (12) [38,39].

- They are conditionally hyperbolic, i.e., hyperbolic only for certain values of $\mathbf{u}$. For some values of $\mathbf{u}$, the matrix $A(\mathbf{u})$ has complex eigenvalues.

- The eigenstructure of $A$ cannot be written down in a simple analytic form.

- They are neither genuinely nonlinear nor linearly degenerate over all of phase space, i.e., smooth waves can break and become discontinuous, but they do not necessarily break.

These properties will be described in more detail in sections 4 and 5 .

Because of these properties, designing a numerical scheme is a challenge. Since the equations are nonconservative, the analytical tools for conservations laws, such as Rankine-Hugoniot jump conditions, do not have unambiguous definitions; and standard numerical methods for conservation laws cannot be readily used [39]. Analysis of nonconservative PDE is an active area of research (see, for instance, [40-43] and references therein), and some methods have been developed to extend Riemann solver-based numerical methods for conservation laws to nonconservative systems (see $[44,45]$ and references therein). However, such methods are difficult to apply here because the eigenstructure of this system is not accessable analytically. For these reasons, the following simple, computationally inexpensive scheme is proposed. 
In short, to solve (13) numerically, the equations are split into three parts: a conservative part, a nonconservative part, and an external forcing part:

$$
\frac{\partial \mathbf{u}}{\partial t}+\frac{\partial}{\partial x} \mathbf{F}(\mathbf{u})=-A_{n c}(\mathbf{u}) \frac{\partial \mathbf{u}}{\partial x}+\mathbf{S}, \quad \frac{\partial \mathbf{F}}{\partial \mathbf{u}}=A_{c} .
$$

The conservative part is solved using a non-oscillatory central scheme [46,47]; the nonconservative part is solved using the method of lines (see [39] and references therein) with centered differences,

$$
\left.\frac{\partial u}{\partial x}\right|_{x=x_{j}} \approx \frac{u_{j+1}-u_{j-1}}{2 \Delta x}
$$

for the spatial derivatives and 2nd-order Runge-Kutta to advance in time; and the external forcing terms will be solved using a 2nd-order Runge-Kutta scheme. Strang splitting is used to combine these three parts into a 2nd-order scheme [39,48].

The splitting $A=A_{c}+A_{n c}$ in (15) can be done in many ways. The choice used here is

$$
\begin{aligned}
\frac{\partial u_{1}}{\partial t}+ & \frac{\partial}{\partial x}\left[-\theta_{1}+\frac{3}{\sqrt{2}} u_{1} u_{2}\right] & =\frac{3}{2 \sqrt{2}} u_{1} \frac{\partial u_{2}}{\partial x}+S_{u_{1}} \\
\frac{\partial \theta_{1}}{\partial t}+ & \frac{\partial}{\partial x}\left[-u_{1}+\sqrt{2} u_{1} \theta_{2}-\frac{1}{\sqrt{2}} u_{2} \theta_{1}\right] & =-\frac{1}{\sqrt{2}}\left[2 \theta_{2} \frac{\partial u_{1}}{\partial x}+\frac{1}{2} \theta_{1} \frac{\partial u_{2}}{\partial x}\right]+S_{\theta_{1}} \\
\frac{\partial u_{2}}{\partial t}+ & \frac{\partial}{\partial x}\left[-\theta_{2}\right] & =S_{u_{2}} \\
\frac{\partial \theta_{2}}{\partial t}+ & \frac{\partial}{\partial x}\left[-\frac{1}{4} u_{2}\right] & =-\frac{1}{2 \sqrt{2}}\left[u_{1} \frac{\partial \theta_{1}}{\partial x}-\theta_{1} \frac{\partial u_{1}}{\partial x}\right]+S_{\theta_{2}}
\end{aligned}
$$

Note that three nonlinear terms appear in the flux on the left-hand side, and the other nonlinear terms appear on the right-hand side. This splitting $A=A_{c}+A_{n c}$ has the following properties:

- The conservative advection matrix $A_{c}(\mathbf{u})$ has eigenvalues $\pm \frac{1}{2}, \frac{1}{\sqrt{2}} u_{2} \pm \sqrt{1+2 u_{2}^{2}-\sqrt{2} \theta_{2}}$, so that the time step of the central scheme can be easily chosen to meet the CFL condition, and the conservative part is hyperbolic for $\theta_{2}<1 / \sqrt{2}$ (which is $\approx 11 \mathrm{~K}$ in dimensional units).

- The nonconservative advection matrix $A_{n c}(\mathbf{u})$ is nilpotent, i.e., the linear wavespeeds of $A_{n c}(\mathbf{u})$ are all zero for all $\mathbf{u}$. This property seems to justify using centered differences in (16) instead of upwind differences.

To test for second-order convergence of this numerical method, a convergence test was carried out with the numerical solution compared to an "exact" simple wave solution. Simple waves are discussed in section 5, and the numerical procedure used to calculate the "exact" solution is described in appendix A. The simple wave used for the convergence test propagates eastward at $50 \mathrm{~m} / \mathrm{s}$ with a background state of $\theta_{1}=4 \mathrm{~K}, u_{1}=u_{2}=\theta_{2}=0$. The initial condition is nearly sinusoidal, and the wave breaks at time $t=2.15$ hours, but the exact and numerical solutions are compared at time $t=2.0$ hours, just before the wave breaks. This is shown in Figure 2. Grid sizes from 32 to 4096 points were tested, and Figure 2 shows a comparison of the exact and numerical solutions for $u_{1}$ for the cases with 32 , 64,128 , and 256 grid points. The case with 256 grid points seems to capture the wave well visually, whereas the cases with fewer grid points have notable discrepancies. Figure 3 shows the results of the convergence test using the $L^{1}$ error:

$$
L^{1} \text { error }=\frac{1}{N} \sum_{i=1}^{N}\left|u_{n u m}(i \Delta x, t)-u_{e x}(i \Delta x, t)\right|,
$$

where $N$ is the number of grid points, $u_{n u m}$ is the numerical solution, and $u_{e x}$ is the "exact" solution. The errors in $\theta_{1}$ and $\theta_{2}$ (not shown) are indistinguishable from those of $u_{1}$ and $u_{2}$ in Figure 3 , respectively. Second-order convergence is demonstrated in each of the variables. 

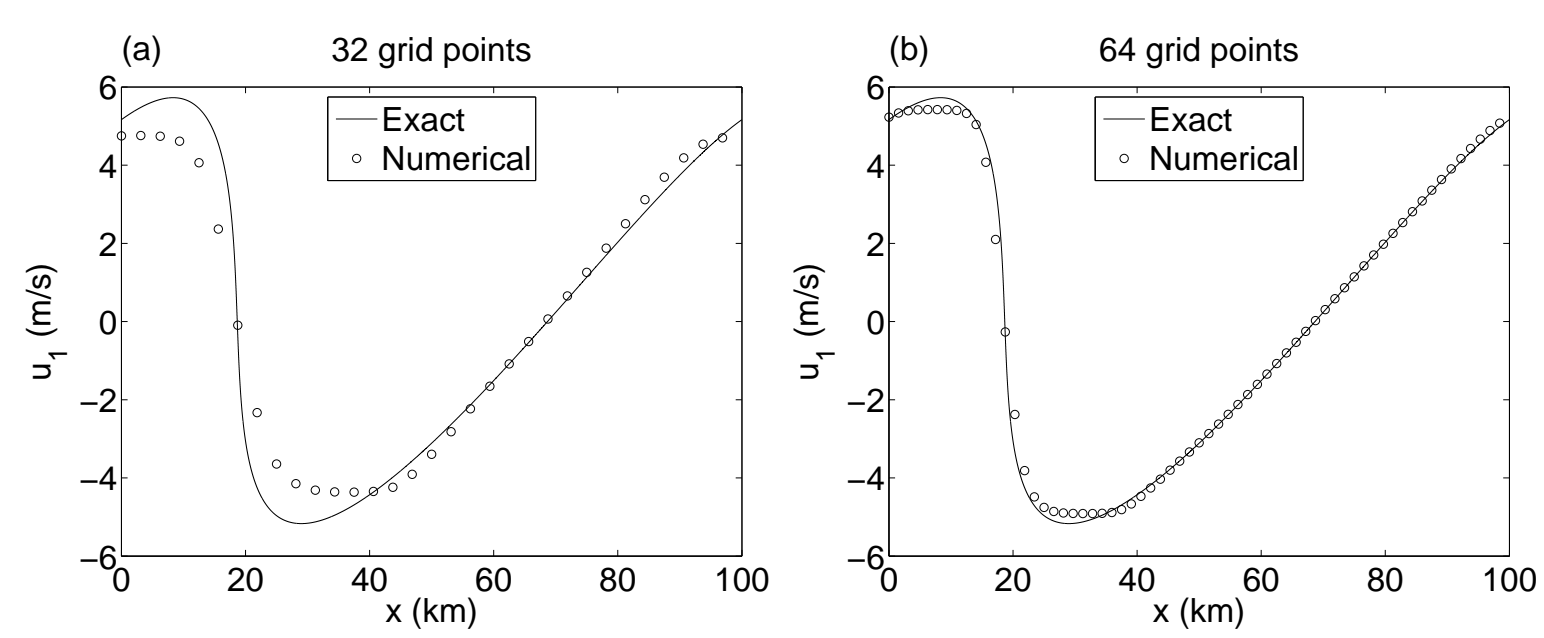

(c)

128 grid points
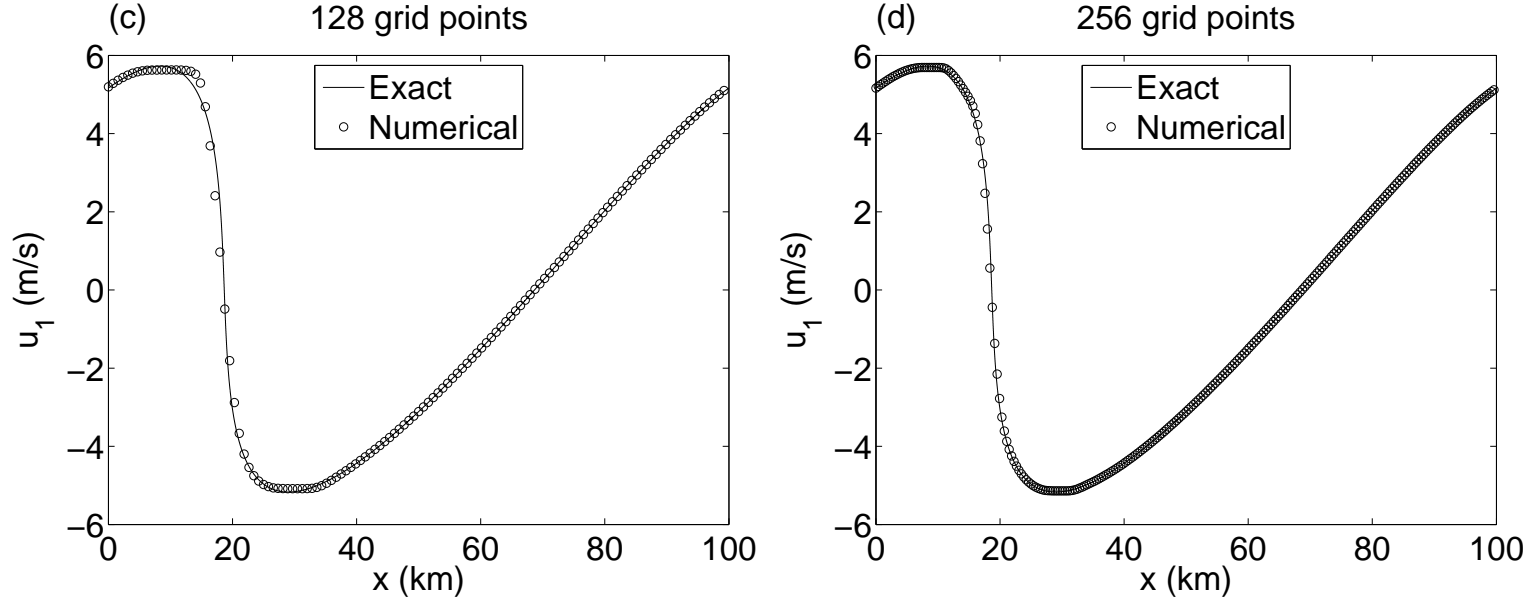

Fig. 2 Comparison of "exact" simple wave solution with numerical solution at time $t=2$ hours for (a) 32 , (b) 64, (c) 128 , and (d) 256 grid points.
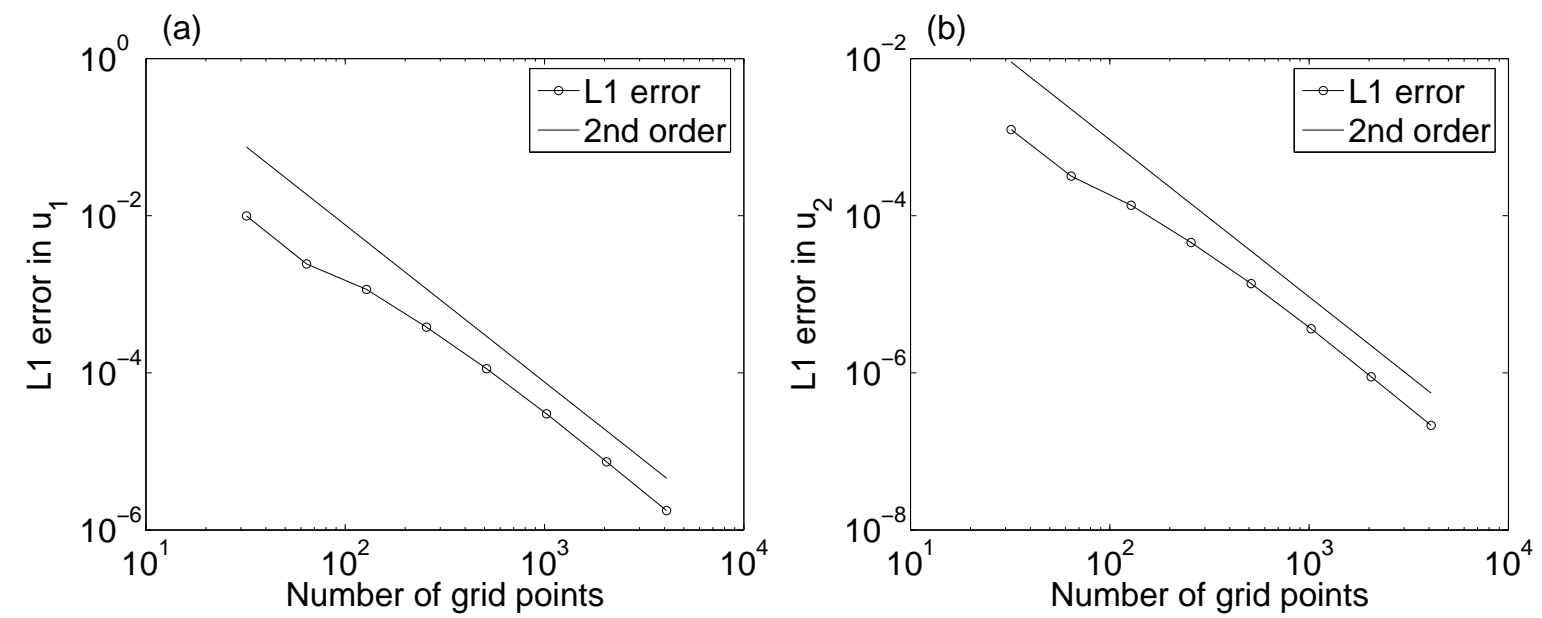

Fig. 3 Convergence test for numerical method compared to an "exact" simple wave solution at time $t=2$ hours as in Figure 2. Grids with 32, 64, ․ 4096 points were used for the test. The plots show the $L^{1}$ error in (a) $u_{1}$ and (b) $u_{2}$ as lines with circles, and a solid line shows the slope of theoretical second-order convergence. The numerical method demonstrates second-order convergence in each of the variables. 


\section{Hyperbolicity}

In this section the linear waves of the 2MSWE are studied for different background states, and it is shown that the equations can become non-hyperbolic in some situations. Illustrations are given of both the hyperbolic travelling waves and the non-hyperbolic overturning waves. Then, using the nonlinear equations, hyperbolicity is examined numerically by forcing the system into non-hyperbolic states using imposed source terms. This test shows that the numerical scheme can handle non-hyperbolic states in a reasonable way.

\subsection{Linear Hyperbolic Waves}

If the 2MSWE (13) are linearized about the constant solution

$$
\mathbf{u}=\left(u_{1}, \theta_{1}, u_{2}, \theta_{2}\right)^{T}=\left(\bar{u}_{1}, \bar{\theta}_{1}, \bar{u}_{2}, \bar{\theta}_{2}\right)^{T},
$$

the resulting linearized equations are

$$
\mathbf{u}_{t}+A(\overline{\mathbf{u}}) \mathbf{u}_{x}=0, \quad \text { where } A(\overline{\mathbf{u}})=\left(\begin{array}{cccc}
\frac{3}{\sqrt{2}} \bar{u}_{2} & -1 & \frac{3}{2 \sqrt{2}} \bar{u}_{1} & 0 \\
-1+2 \sqrt{2} \bar{\theta}_{2} & -\frac{1}{\sqrt{2}} \bar{u}_{2} & -\frac{1}{2 \sqrt{2}} \bar{\theta}_{1} & \sqrt{2} \bar{u}_{1} \\
0 & 0 & 0 & -1 \\
-\frac{1}{2 \sqrt{2}} \bar{\theta}_{1} & \frac{1}{2 \sqrt{2}} \bar{u}_{1} & -\frac{1}{4} & 0
\end{array}\right) .
$$

Linear equations of this form have only nondispersive solutions (see chapter 5 of [1]), which take the form of travelling wave solutions $\mathbf{u}(x, t)=\mathbf{r} f(x-\lambda t)$. If this ansatz is inserted into (19), an eigenvalue problem is obtained for the wave speeds $\lambda$ and the eigenvectors $\mathbf{r}$ :

$$
[-\lambda I+A(\overline{\mathbf{u}})] \mathbf{r}=0 .
$$

Table 2 lists $\lambda$ and $\mathbf{r}$ for several choices of $\overline{\mathbf{u}}$. In general, $\bar{u}_{2}$ and $\bar{\theta}_{2}$ have a much stronger effect than $\bar{u}_{1}$ and $\bar{\theta}_{1}$. The strongest effect occurs with $\bar{\theta}_{2}=+2.5 \mathrm{~K}$, for which the 1 st baroclinic wave speeds decrease in magnitude from $\pm 50 \mathrm{~m} / \mathrm{s}$ to $\pm 36 \mathrm{~m} / \mathrm{s}$. Notice that the eigenvector is either entirely in mode- 1 or entirely in mode- 2 unless there is a mode- 1 background state. This can be seen from the form of $A(\mathbf{u})$ in (19).

Plots of the waves for a trivial background state $\bar{u}_{1}=\bar{u}_{2}=\bar{\theta}_{1}=\bar{\theta}_{2}=0$ (corresponding to the first two linear baroclinic equations in (7) in 1D, without rotation) are shown in Figure 4. These are the first four waves listed in Table 2 . The mode- 1 waves propagate at speeds of $\pm 50 \mathrm{~m} / \mathrm{s}$, and the mode- 2 waves propagate at speeds of $\pm 25 \mathrm{~m} / \mathrm{s}$.

\subsection{Linear Non-Hyperbolic Waves}

Here it is shown that the 2MSWE are conditionally hyperbolic, i.e., they are hyperbolic in some regions of phase space and non-hyperbolic in other regions. The system in (19) is hyperbolic if the $4 \times 4$ matrix $A(\overline{\mathbf{u}})$ has real eigenvalues and linearly independent eigenvectors. Finding the eigenvalues for the general matrix $A(\overline{\mathbf{u}})$ is not analytically tractable. Instead, the eigenvalues and eigenvectors of $A(\overline{\mathbf{u}})$ are computed numerically for a representative set of fixed background states. First consider four simple background states where only one of $\left(u_{1}, u_{2}, \theta_{1}, \theta_{2}\right)$ is nonzero:
1. $u_{1} \neq 0, u_{2}=\theta_{1}=\theta_{2}=0$. The eigenvalues are all real unless $\left|u_{1}\right|>\sqrt{2 / 3}$
2. $\theta_{1} \neq 0, u_{1}=u_{2}=\theta_{2}=0$. The eigenvalues are all real unless $\left|\theta_{1}\right|>\sqrt{2}$
$(41 \mathrm{~m} / \mathrm{s})$.
3. $u_{2} \neq 0, u_{1}=\theta_{1}=\theta_{2}=0$. The eigenvalues are all real.
4. $\theta_{2} \neq 0, u_{1}=u_{2}=\theta_{1}=0$. The eigenvalues are all real unless $\theta_{2}>1 /(2 \sqrt{2})$

These results suggest a sufficient condition for the 2MSWE system to lose hyperpolicity is that either the background shear $\left|u_{1}\right|$ or $\left|\theta_{1}\right|$ becomes too large. Large jet shears from $\left|u_{2}\right|$, however, will not affect 
Table 2 Linear waves of 2MSWE for different background states. The first four columns show the background state used. The middle column lists the four linear wavespeeds $\lambda$. The last four columns show the eigenvector $\mathbf{r}$ corresponding to each wavespeed $\lambda$.

\begin{tabular}{|c|c|c|c|c|c|c|c|c|}
\hline \multirow[b]{2}{*}{$\bar{u}_{1}(\mathrm{~m} / \mathrm{s})$} & \multicolumn{3}{|c|}{ Background state } & \multirow{2}{*}{$\begin{array}{r}\lambda \\
(\mathrm{m} / \mathrm{s})\end{array}$} & \multicolumn{4}{|c|}{ Eigenvector $\mathbf{r}$ (nondim.) } \\
\hline & $\bar{\theta}_{1}(\mathrm{~K})$ & $\bar{u}_{2}(\mathrm{~m} / \mathrm{s})$ & $\bar{\theta}_{2}(\mathrm{~K})$ & & $u_{1}$ & $\theta_{1}$ & $u_{2}$ & $\theta_{2}$ \\
\hline \multirow[t]{4}{*}{0} & 0 & 0 & 0 & -50.0 & 0.71 & 0.71 & 0 & \\
\hline & & & & -25.0 & & & 0.89 & 0.45 \\
\hline & & & & +25.0 & 0 & 0 & 0.89 & -0.45 \\
\hline & & & & +50.0 & 0.71 & -0.71 & 0 & 0 \\
\hline \multirow[t]{4}{*}{+5} & 0 & 0 & 0 & -50.3 & 0.71 & 0.71 & -0.03 & -0.03 \\
\hline & & & & -24.7 & 0.14 & 0.16 & 0.88 & 0.43 \\
\hline & & & & +24.7 & -0.14 & 0.16 & 0.88 & -0.43 \\
\hline & & & & +50.3 & 0.71 & -0.71 & 0.03 & -0.03 \\
\hline \multirow[t]{4}{*}{0} & +5 & 0 & 0 & -50.5 & 0.70 & 0.70 & 0.11 & 0.11 \\
\hline & & & & -24.1 & -0.14 & -0.07 & 0.89 & 0.43 \\
\hline & & & & +24.1 & -0.14 & 0.07 & 0.89 & -0.43 \\
\hline & & & & +50.5 & 0.70 & -0.70 & 0.11 & -0.11 \\
\hline \multirow[t]{4}{*}{0} & 0 & +5 & 0 & -47.0 & 0.66 & 0.76 & 0 & 0 \\
\hline & & & & -25.0 & 0 & 0 & 0.89 & 0.45 \\
\hline & & & & +25.0 & 0 & 0 & 0.89 & -0.45 \\
\hline & & & & +54.0 & 0.76 & -0.66 & 0 & 0 \\
\hline \multirow[t]{4}{*}{0} & 0 & 0 & +2.5 & -36.4 & 0.81 & 0.59 & 0 & 0 \\
\hline & & & & -25.0 & & 0 & 0.89 & 0.45 \\
\hline & & & & +25.0 & 0 & 0 & 0.89 & -0.45 \\
\hline & & & & +36.4 & 0.81 & -0.59 & 0 & 0 \\
\hline
\end{tabular}

hyperbolicity, and neither will strongly negative $\theta_{2}$, but strongly positive $\theta_{2}$ will make the system lose its hyperbolicity.

When hyperbolicity is lost, at least one of the eigenvectors has a positive growth rate. Plots of the unstable waves for the four cases in (21) are shown in Figure 5. For large positive $\bar{\theta}_{1}$, there is downward transport of cold air and upward transport of warm air. This overturning circulation should lead to a more stable stratification. Note that this unstable wave has its strongest amplitude at upper levels; this is because large positive $\bar{\theta}_{1}$ leads to a less stable stratification at upper levels and a more stable stratification at lower levels (see Figure 1). For similar reasons, large negative $\bar{\theta}_{1}$ creates an instability at lower levels, as shown in Figure $5 \mathrm{~b}$, and large positive $\bar{\theta}_{2}$ creates an instability at middle levels, as shown in Figure $5 \mathrm{c}$. Notice from (21) that for large negative $\bar{\theta}_{2}$ the 2MSWE are hyperbolic, and there is a more stable stratification at middle levels. On the other hand, this case of large negative $\bar{\theta}_{2}$ also leads to a less stable stratification at the lowest and highest levels (see Figure 1), but, interestingly, the 2MSWE remain hyperbolic for this case. This is possibly due to the crude vertical resolution of the 2MSWE, which cannot resolve a wave in the shallow unstably stratified layer that appears when $\bar{\theta}_{2}$ takes large negative values. It would be interesting to see what would happen if more baroclonic modes were retained. Also note that the unstable waves in Figure 5 are all stationary, i.e., they all have zero phase speed. This is not necessarily the case when more than one background state variable are not zero, as seen below in Table 3. Finally, note that the dispersion relation for (19) is $\omega(k)=\lambda k$. Therefore, for the unstable waves in Figure 5, which grow exponentially in time, the smallest scales (largest $k$ ) have the fastest growth rates. In atmospheric science, this type of behavior is seen, for instance, with conditional instability of the second kind (CISK) in convergence-based convective parametrizations [3, 49].

When two of $\bar{u}_{1}, \bar{\theta}_{1}, \bar{u}_{2}, \bar{\theta}_{2}$ are allowed to be nonzero at the same time, the hyperbolic region of state space becomes harder to describe, as shown in Figure 6. In these plots, hyperbolic regions (labelled as "hyp.") and non-hyperbolic regions (labelled as "non-hyp.") are separated by the solid curves. While large values of $\left|u_{1}\right|,\left|\theta_{1}\right|$, and $\theta_{2}$ tend to cause non-hyperbolicity, large values of $\left|u_{2}\right|$ and strongly negative $\theta_{2}$ tend to maintain hyperbolicity. An example of the complexity of the hyperbolic region is the case of Figure 6e with nonzero $\bar{u}_{1}$ and $\bar{\theta}_{2}$. In that case some hyperbolic regions appear for large values of $\left|u_{1}\right|$ and values of $\theta_{2}$ in the range $\approx 5-7 \mathrm{~K}$.

Since the shear and stratification determine the hyperbolicity of the 2MSWE, one might expect there to be a Richardson number criterion for hyperbolicity. The Richardson number is the ratio of 
(a) Phase speed $=-50 \mathrm{~m} / \mathrm{s}$

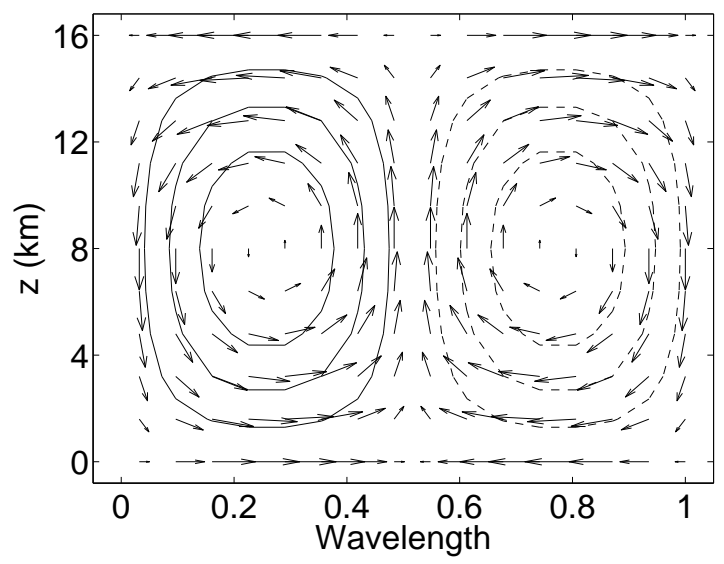

(c) Phase speed $=-25 \mathrm{~m} / \mathrm{s}$

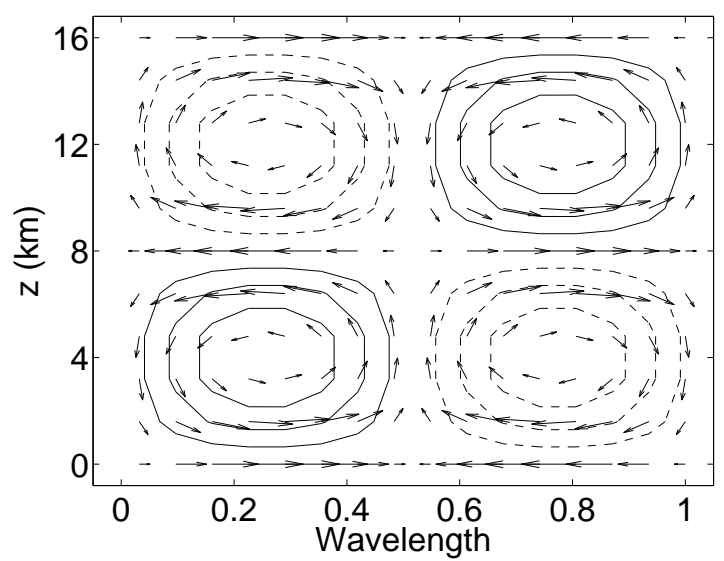

(b) Phase speed $=+50 \mathrm{~m} / \mathrm{s}$

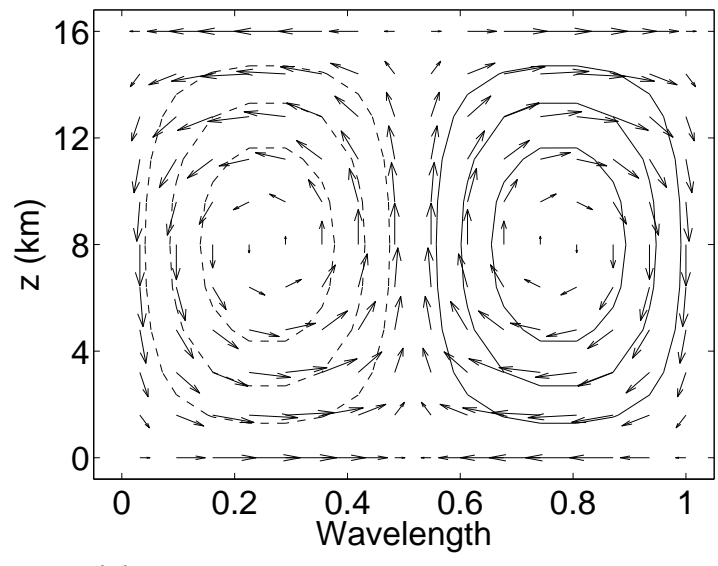

(d) Phase speed $=+25 \mathrm{~m} / \mathrm{s}$

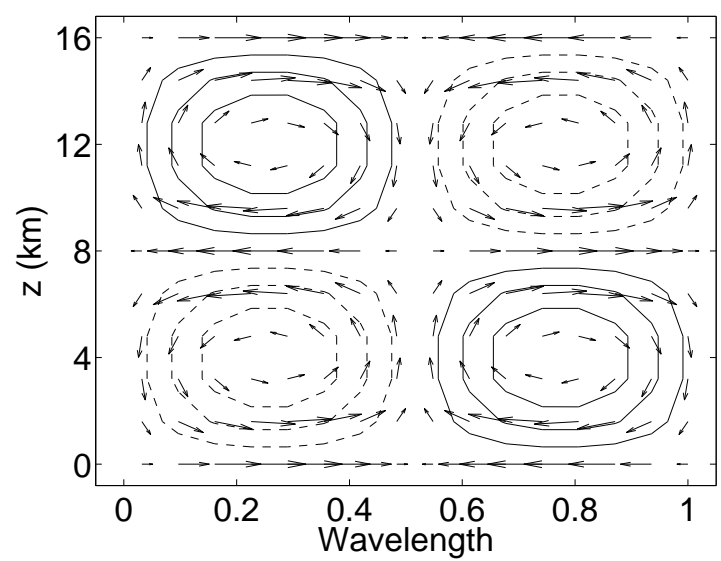

Fig. 4 Linear waves of 2 MSWE for the trivial background state $\bar{u}_{1}=\bar{u}_{2}=\bar{\theta}_{1}=\bar{\theta}_{2}=0$. The waves have phase speeds of (a) -50 , (b) +50 , (c) -25 , and (d) $+25 \mathrm{~m} / \mathrm{s}$. Solid contours denote positive anomalies of potential temperature, and dashed contours denote negative anomalies.

the stratification to vertical wind shear squared $[1,3]$ :

$$
R i=\frac{N_{\text {total }}^{2}}{(\partial U / \partial z)^{2}}, \quad N_{\text {total }}^{2}=\frac{g}{\theta_{\text {ref }}} \frac{\partial \theta_{\text {total }}}{\partial z} .
$$

With the truncated vertical structure of the 2MSWE, a Richardson number could be defined as

$$
R i=\frac{f\left(\theta_{1}, \theta_{2}\right)}{g\left(u_{1}, u_{2}\right)},
$$

where $f$ and $g$ are functions to be determined. However, given the results in this section, it is not clear how $f$ and $g$ should be defined. Ultimately the hyperbolicity of the 2MSWE is determined by the roots of the characteristic polynomial of $A(\overline{\mathbf{u}})$ of $(19)$ :

$$
\begin{aligned}
\lambda^{4}- & \sqrt{2} u_{2} \lambda^{3}+\frac{1}{4}\left(-5+8 \sqrt{2} \theta_{2}-2 u_{1}^{2}-6 u_{2}^{2}\right) \lambda^{2} \\
& +\frac{1}{2 \sqrt{2}}\left(-2 \sqrt{2} u_{1} \theta_{1}+u_{2}+3 u_{1}^{2} u_{2}\right) \lambda+\frac{1}{8}\left(2-\theta_{1}^{2}-4 \sqrt{2} \theta_{2}-3 u_{1}^{2}+6 \sqrt{2} u_{1}^{2} \theta_{2}+3 u_{2}^{2}\right) .
\end{aligned}
$$

This polynomial, though, is no more analytically tractable than an analytic Richardson number criterion. Nevertheless, the results in this section suggest empirical conditions for hyperbolicity that are physically intuitive. 
(a)
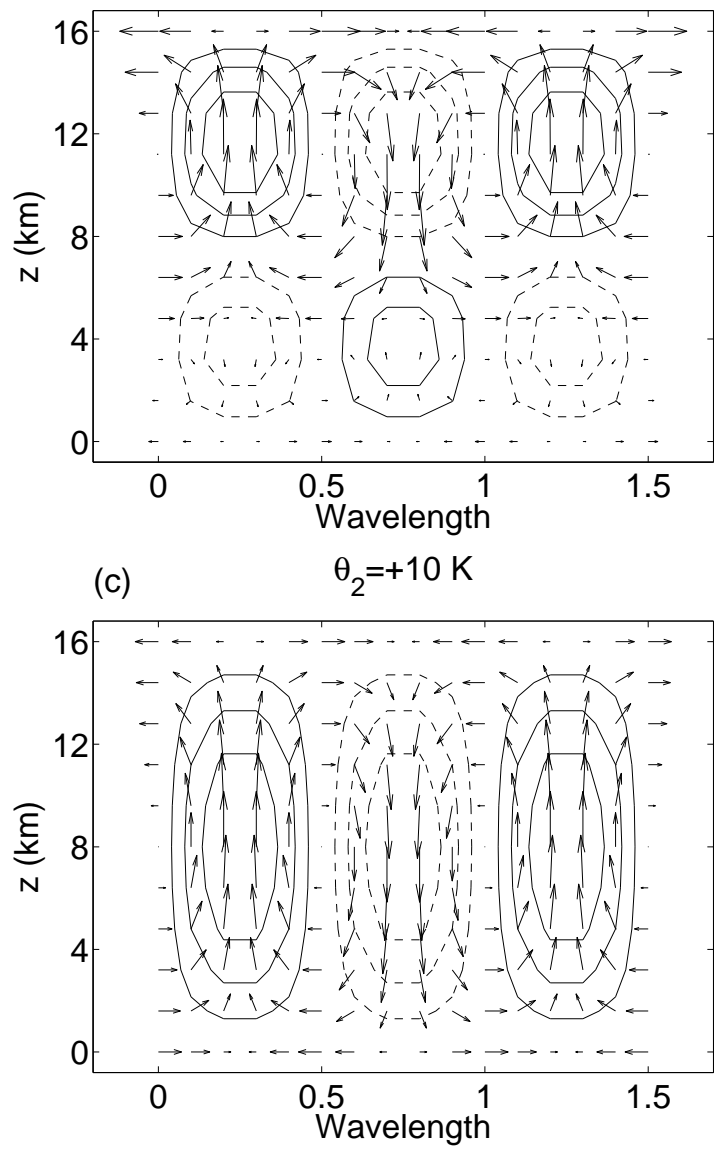

(b) $\quad \theta_{1}=-30 \mathrm{~K}$
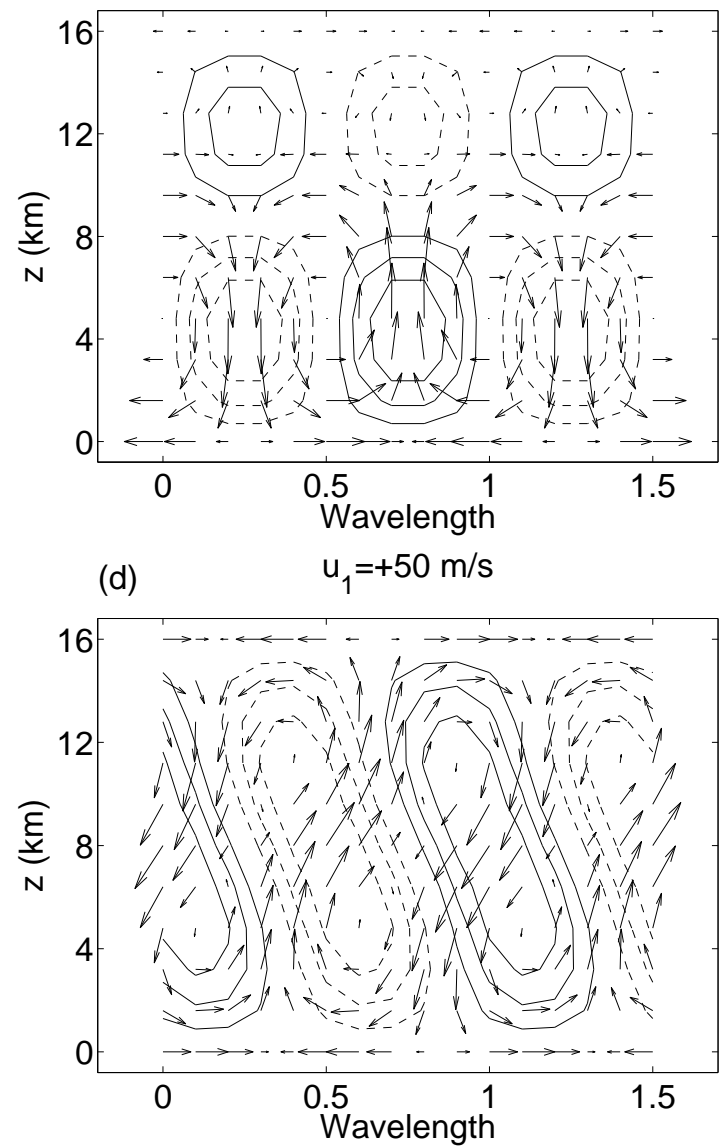

Fig. 5 Unstable waves for various non-hyperbolic background states. In each case, only one of $\bar{u}_{1}, \bar{u}_{2}, \bar{\theta}_{1}, \bar{\theta}_{2}$ is nonzero: (a) $\bar{\theta}_{1}=+30 \mathrm{~K}$, (b) $\bar{\theta}_{1}=-30 \mathrm{~K}$, (c) $\bar{\theta}_{2}=+10 \mathrm{~K}$, and (d) $\bar{u}_{1}=+50 \mathrm{~m} / \mathrm{s}$. Solid contours are positive anomalies of potential temperature, and dashed contours are negative anomalies.

\subsection{Forcing the System into Non-Hyperbolic States}

If the system enters a state $\left(u_{1}, \theta_{1}, u_{2}, \theta_{2}\right)$ that is non-hyperbolic, it could potentially be problematic for the numerical scheme. This might happen if source terms are included (e.g., the effects of water vapor and clouds as in [18-23]) and the source terms become too strong. To test how the numerical scheme behaves when non-hyperbolic states are reached, imposed source terms are used to force the system into non-hyperbolic states. It is shown that the numerical scheme responds in a physically reasonable way.

The imposed forcing is chosen to represent condensational heating from deep convective and congestus clouds, which are observed to directly force the 1st and 2nd baroclinic modes, respectively (see [18] and references therein). For simplicity, the forcing is assumed to be localized in space and periodic in time:

$$
\begin{aligned}
& S_{\theta 1}(x, t)=H(t) a \exp \left(-\frac{\left(x-x_{0}\right)^{2}}{2 \sigma^{2}}\right), \quad H(t)=\left\{\begin{array}{lll}
1 & \text { for } & \bmod (t, 12 \mathrm{hr})<4 \mathrm{hr} \\
0 & \text { for } \quad \bmod (t, 12 \mathrm{hr})>4 \mathrm{hr}
\end{array}\right. \\
& S_{\theta 2}(x, t)=\frac{1}{2} S_{\theta 1}
\end{aligned}
$$

where the heating is centered in the middle of the domain at $x_{0}=1000 \mathrm{~km}$, the standard deviation is $\sigma=20 \mathrm{~km}$, and the amplitude is $a=200 \mathrm{~K} /$ day $\approx 8 \mathrm{~K} /$ hour. This is a typical heating rate for the deep convective region of a squall line or mesoscale convective system $[9,53]$. 
(a)
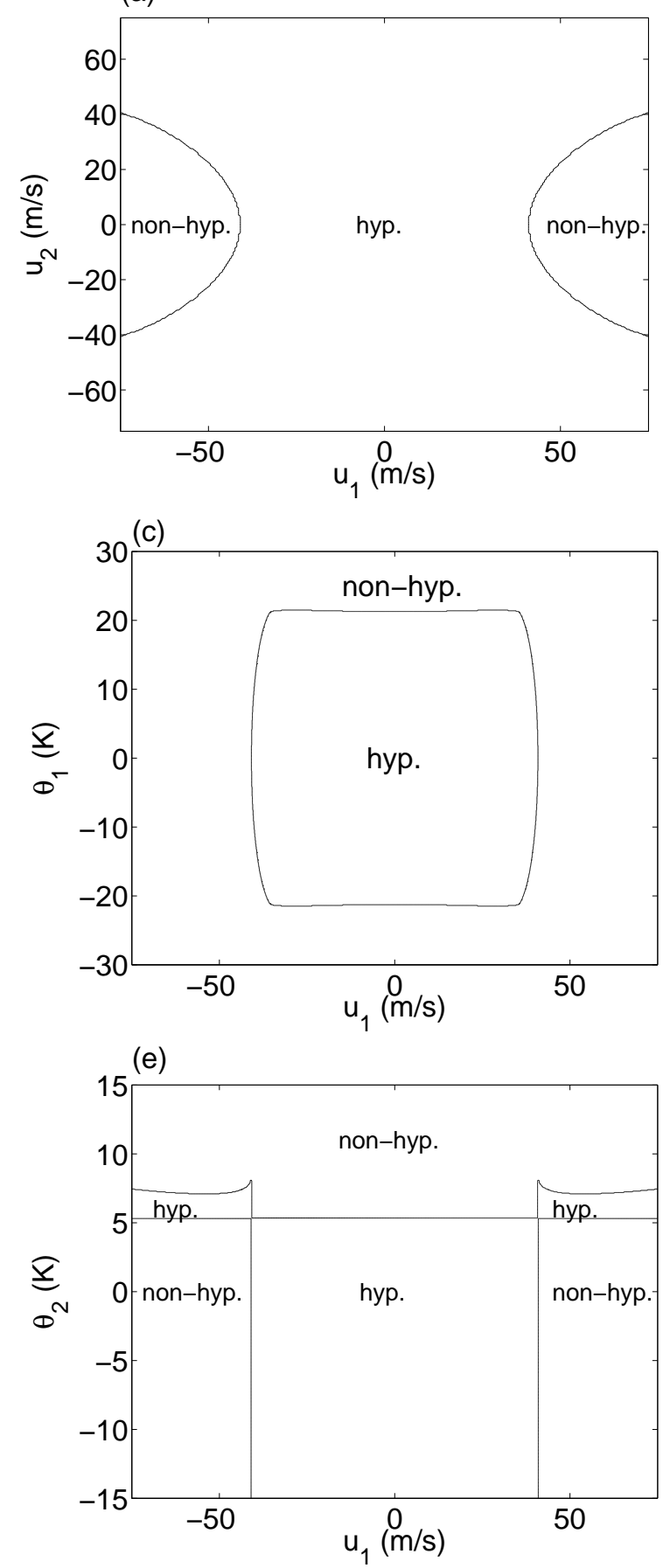

(b)
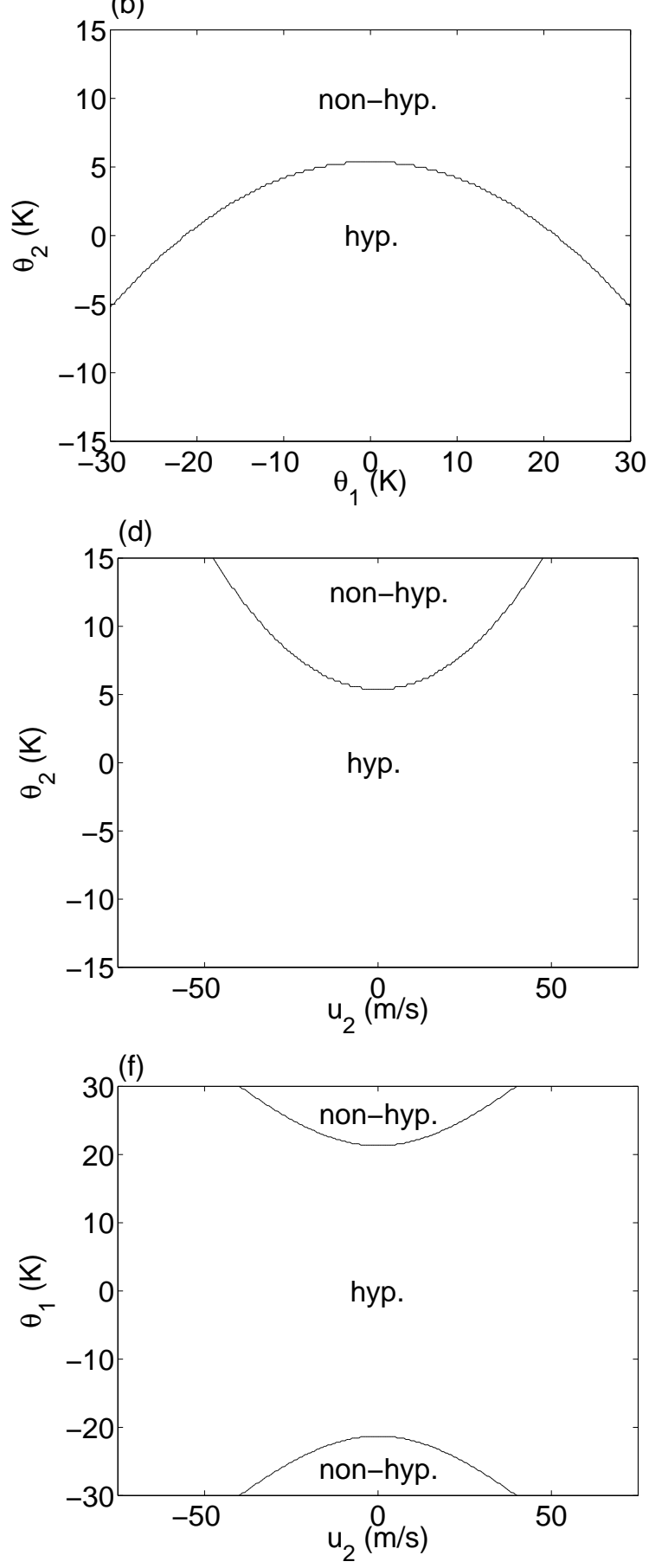

Fig. 6 Hyperbolic and non-hyperbolic regions of state space for different background states. For each case, two variables are set to zero and the other two are varied, as shown: (a) $u_{1}$ and $u_{2}$, (b) $\theta_{1}$ and $\theta_{2}$, (c) $u_{1}$ and $\theta_{1},(\mathrm{~d}) u_{2}$ and $\theta_{2}$, (e) $u_{1}$ and $\theta_{2}$, and (f) $u_{2}$ and $\theta_{1}$. 
Table 3 Some sample points where hyperbolicity was lost in the simulation in Figure 7

\begin{tabular}{ll|rrrrr|rrr}
\hline \multicolumn{2}{c|}{ Location } & \multicolumn{5}{c|}{ State } & \multicolumn{2}{|l}{ Eigenvalues $(\mathrm{m} / \mathrm{s})$} \\
$x(\mathrm{~km})$ & $t(\mathrm{hr})$ & $u_{1}(\mathrm{~m} / \mathrm{s})$ & $\theta_{1}(\mathrm{~K})$ & $u_{2}(\mathrm{~m} / \mathrm{s})$ & $\theta_{2}(\mathrm{~K})$ & & \\
\hline 950 & 25 & 8.4 & 6.5 & 2.8 & 3.1 & 43, & 17, & $-28 \pm 1.6 i$ \\
400 & 44 & 19.3 & 6.6 & -3.2 & 3.6 & 44, & 8, & $-29 \pm 8 i$ \\
\hline
\end{tabular}

The mode- 1 and -2 heatings have the same strength when their vertical structures are considered, since the basis functions for $\theta_{1}$ and $\theta_{2}$ are $\sqrt{2} \sin (\pi z)$ and $2 \sqrt{2} \sin (2 \pi z)$, respectively. This heating is applied for 4 hours and then turned off for 8 hours, and this process is repeated periodically for the simulation duration of 24 days. The grid spacing is $\Delta x=2 \mathrm{~km}$ on a $2000 \mathrm{~km}$-wide domain. The initial conditions are $u_{1}=\theta_{1}=u_{2}=\theta_{2}=0$.

Figure 7a shows the time evolution of the total energy. Initially the energy evolves in a stair-step pattern, increasing as the source terms are turned on and remaining constant while the source terms are turned off. The stair-step pattern lasts less than 2 days, after which the system dissipates energy at the same time the forcing pumps energy into the system. Figure $7 \mathrm{~b}$ shows the time evolution of the domain-maximum of $\theta_{2}$. Note that $\theta_{2}$ is bounded above by $\approx 6 \mathrm{~K}$, which was the simple criterion for hyperbolicity given in (21). Figure $7 \mathrm{c}$ shows a black square at each point $(x, t)$ where hyperbolicity is lost. Non-hyperbolic states are common in this simulation. The results in Figure 7 suggest that the source terms force $\theta_{2}$ to increase until non-hyperbolic states are reached, and the unstable states (as in Figure 5) act to stabilize the system and bring it back to hyperbolic states with $\theta_{2}<6 \mathrm{~K}$. The energy plot in Figure 7a shows that the non-hyperbolic unstable waves do not cause catastrophic problems for the numerical scheme, since the numerical solution does not grow without bound. Note also that while the 2MSWE became non-hyperbolic, the conservative part of the numerical scheme is always hyperbolic, since it is non-hyperbolic only when $\theta_{2}>11 \mathrm{~K}$, as described in section 3 .

Some sample points where hyperbolicity was lost are shown in Table 3 . These sample states have nonzero contributions from each variable. Each individual contribution is well within the limits of hyperbolicity that were described in (21) and Figure 6, but taken together they lead to a non-hyperbolic state. Therefore, while the results in (21) and Figure 6 suggest that relatively large values must be reached in order for hyperbolicity to be lost, it is not excluded that moderate values can lead to nonhyperbolic states when several of the variables are nonzero. From the different shapes in Figure 6, it is not hard to imagine that the system can become non-hyperbolic through more complex configurations of the background state variables.

\section{Breaking Waves}

In this section, nonlinear solutions to the 2MSWE are studied. It will be shown analytically that smooth initial conditions can break, but there are also exact solutions that do not break. Whether or not a wave breaks depends on the background state; that is, the characteristic fields of the 2MSWE are neither genuinely nonlinear nor linearly degenerate over all of phase space. The breaking waves resemble internal bores [24-27]. The numerical scheme is also tested with breaking waves. After the wave breaks, the numerical front is sharp and without oscillations, and it continues to propagate and decay as an $\mathrm{N}$-wave $[38,39]$. A numerical solution of the dam-break problem demonstrates that the numerical scheme can represent rarefaction waves as well as linearly degenerate fronts and genuinely nonlinear fronts.

\subsection{Simple Wave Solutions}

Now consider the nonlinear 1D 2MSWE (13) written in a quasi-linear form,

$$
\mathbf{u}_{t}+A(\mathbf{u}) \mathbf{u}_{x}=0,
$$

where $\mathbf{u} \equiv\left(u_{1}, \theta_{1}, u_{2}, \theta_{2}\right)$ is a vector solution and $A(\mathbf{u})$ is the corresponding advecting matrix. Here we seek simple wave solution on the form $[38,50]$

$$
\mathbf{u}(x, t)=\mathbf{U}(\sigma(x, t)) .
$$




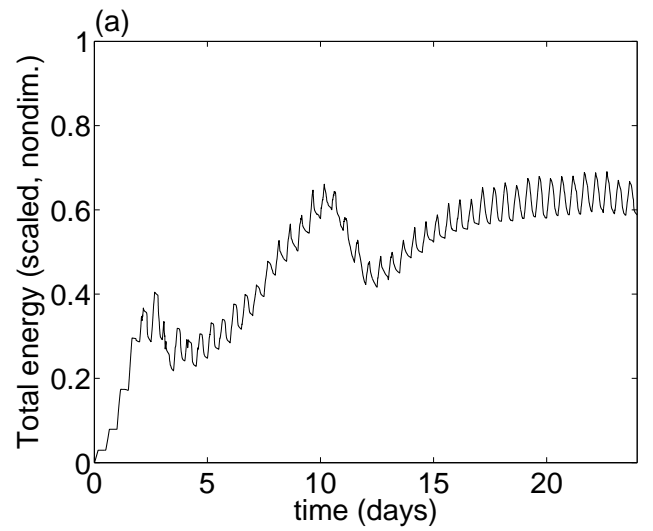

(b)

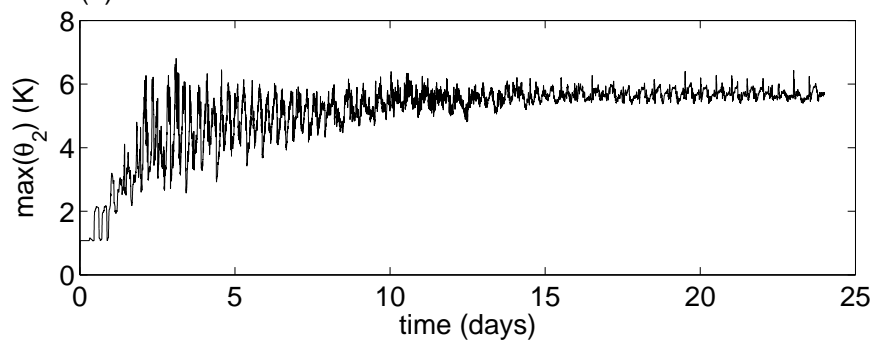

(c) White $=$ Hyperbolic, Black $=$ Not Hyperbolic

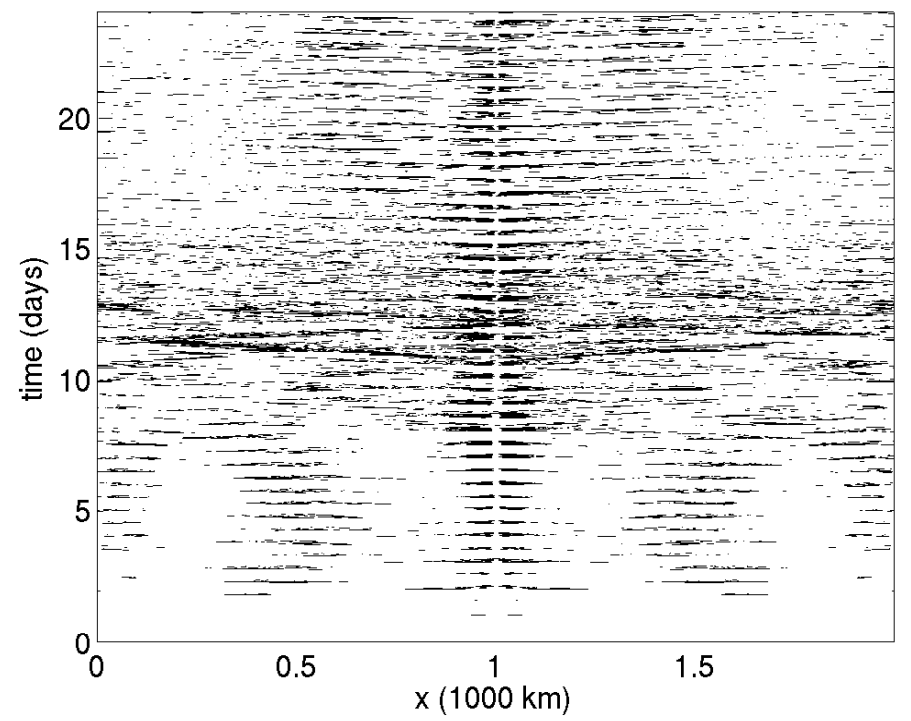

Fig. 7 Results for a simulation with the imposed source terms (23) that force the system into non-hyperbolic states. (a) Time evolution of the total energy. (b) Time evolution of the domain-maximum of $\theta_{2}$, which never exceeds $\approx 6 \mathrm{~K}$. (c) Points $(x, t)$ where hyperbolicity was lost. White squares represent hyperbolic points and black squares represent non-hyperbolic points.

Inserting this ansatz into (24) leads to

$$
\sigma_{t} \mathbf{U}_{\sigma}+\sigma_{x} A(\mathbf{U}(\sigma)) \mathbf{U}_{\sigma}=0
$$

The ansatz (25) will then provide a solution if

$$
\begin{aligned}
\mathbf{U}_{\sigma} & =\mathbf{r}_{p}(\mathbf{U}(\sigma)) \\
\mathbf{U}\left(\sigma_{\text {init }}\right) & =\mathbf{U}_{\text {init }}
\end{aligned}
$$


and

$$
\begin{aligned}
\sigma_{t}+\lambda_{p}(\mathbf{U}(\sigma)) \sigma_{x} & =0 \\
\sigma(x, 0) & =\sigma_{0}(x),
\end{aligned}
$$

where $\mathbf{r}_{p}$ and $\lambda_{p}$ are the eigenvector and eigenvalue corresponding to the $p$ th characteristic field. The ordinary differential equation (ODE) in (26) can be solved on some interval $\sigma_{-}<\sigma<\sigma_{+}$, within which the initial data $\sigma_{0}(x)$ should be chosen. The PDE (27) can then be solved using characteristics:

$$
\frac{d \sigma}{d t}=0 \text { along } \frac{d x}{d t}=\lambda_{p}(\mathbf{U}(\sigma))
$$

which are straight lines. It is assumed in this construction that the sytem remains hyperbolic.

The solution is then

$$
\sigma(x, t)=\sigma_{0}\left(x-\lambda_{p}(\mathbf{U}(\sigma)) t\right)=\sigma_{0}(\alpha)
$$

This solution is valid until two characteristic lines meet, which will happen at time

$$
T_{*}=\frac{-1}{\min _{x} \frac{d}{d x} \lambda_{p}\left(\mathbf{U}\left(\sigma_{0}(x)\right)\right)}=\frac{-1}{\min _{x}\left[\left(\mathbf{r}_{p} \cdot \nabla \lambda_{p}\right) \frac{d \sigma_{0}}{d x}\right]} .
$$

Note that the simple wave will never break if an initial state is chosen so that $\mathbf{r}_{p} \cdot \nabla \lambda_{p}=0$.

Since the wave-breaking condition (30) depends on whether $\mathbf{r}_{p} \cdot \nabla \lambda_{p}$ is nonzero, it is useful to define two common cases: genuine nonlinearity and linear degeneracy [38,50]. For a PDE of the form (24), the $p$ th characteristic field is said to be genuinely nonlinear in an open set $\mathcal{D}$ if

$$
\mathbf{r}_{p}(\mathbf{u}) \cdot \nabla_{u} \lambda_{p}(\mathbf{u}) \neq 0 \text { for all } \mathbf{u} \in \mathcal{D}
$$

For a scalar conservation law $u_{t}+f(u)_{x}=0$, this is the convexity condition $f^{\prime \prime}(u)>0$ (or the concavity condition $\left.f^{\prime \prime}(u)<0\right)$. At the other extreme, the $p$ th characteristic field is said to be linearly degenerate in an open set $\mathcal{D}$ if

$$
\mathbf{r}_{p}(\mathbf{u}) \cdot \nabla_{u} \lambda_{p}(\mathbf{u})=0 \text { for all } \mathbf{u} \in \mathcal{D}
$$

This condition is satisfied trivially by constant-coefficient linear hyperbolic systems, for which $\nabla_{u} \lambda_{p}=0$ for all u. Furthermore, (32) is sometimes satisfied for nonlinear systems; for instance, in gas dynamics, vorticity waves and entropy waves are linearly degenerate [50].

The 2MSWE are neither genuinely nonlinear nor linearly degenerate over all of phase space. To demonstrate this, it is shown that, for each of the characteristic fields $p, \mathbf{r}_{p} \cdot \nabla \lambda_{p}$ is nonzero over parts of phase space and zero over other parts of phase space. Since analytic experssions are not known for $\mathbf{r}_{p}$ and $\lambda_{p}, \mathbf{r}_{p} \cdot \nabla \lambda_{p}$ is calculated numerically. Figure 8 shows plots of $\mathbf{r}_{p} \cdot \nabla \lambda_{p}$ for different points of phase space. Figure 8 a uses points where $u_{2}=\theta_{1}=\theta_{2}=0$ with different values of $u_{1}$. Note that the states shown in this figure are all hyperbolic, which is clear for Figure 8a from the results in (21). The characteristic fields are labelled $p=-1,-2,+2,+1$, where the $+(-)$ corresponds to an eastward (westward) phase speed $\lambda_{p}$, and 1 and 2 refer to whether the eigenvector $\mathbf{r}_{p}$ is predominantly in the 1 st or 2nd baroclinic mode. Figure $8 \mathrm{a}$ shows that none of the characteristic fields is genuinely nonlinear over all of phase space, since $\mathbf{r}_{p} \cdot \nabla \lambda_{p}=0$ for all fields when $u_{1}=\theta_{1}=u_{2}=\theta_{2}=0$. It is also clear from this plot that none of the characteristic fields is linearly degenerate over all of phase space, since $\mathbf{r}_{p} \cdot \nabla \lambda_{p} \neq 0$ for most of the points shown. Figure $8 \mathrm{~b}$ shows that the trivial state $u_{1}=\theta_{1}=u_{2}=\theta_{2}=0$ is not the only point in phase space where $\mathbf{r}_{p} \cdot \nabla \lambda_{p}=0$. This plot shows states with $u_{2}=0, \theta_{1}=4$ $\mathrm{K}, \theta_{2}=2 \mathrm{~K}$, and different values of $u_{1}$. Also note that, for each of the characteristic fields, $\mathbf{r}_{p} \cdot \nabla \lambda_{p}$ is sometimes positive and sometimes negative. Furthermore, while $\mathbf{r}_{p} \cdot \nabla \lambda_{p}=0$ sometimes, there do not appear to be any open sets in phase space where this is true. 

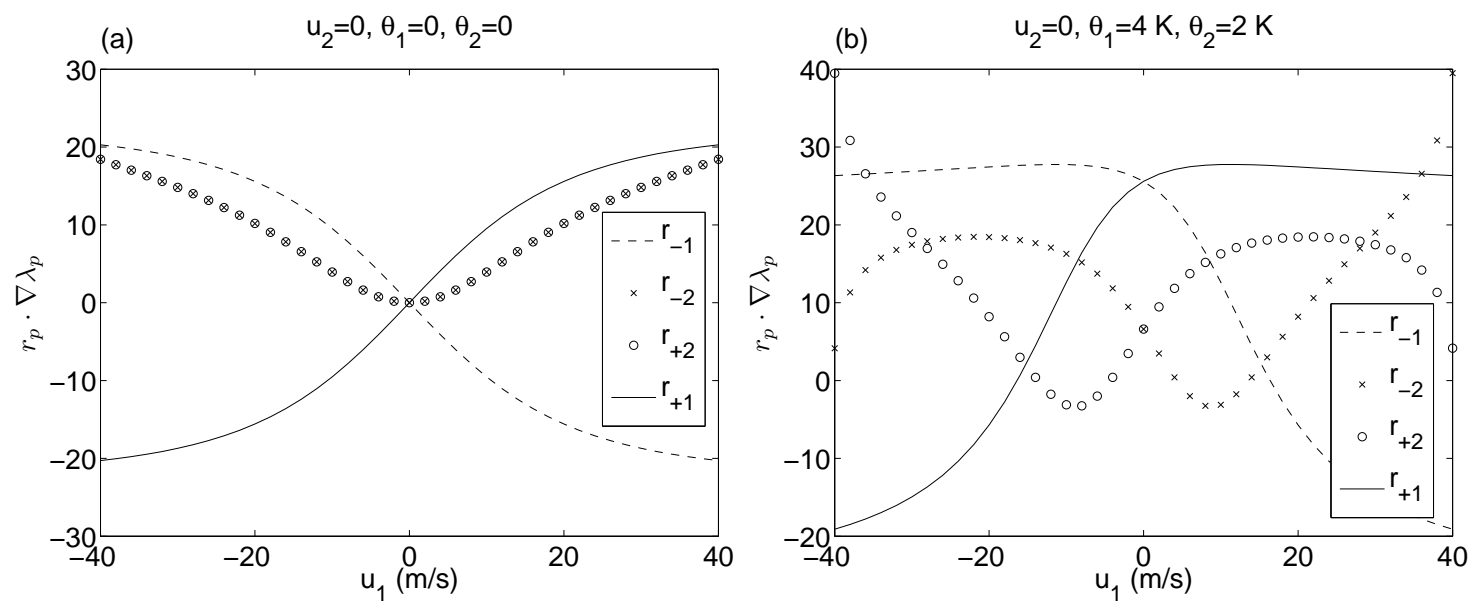

Fig. 8 Plots of $\mathbf{r}_{p}(\mathbf{u}) \cdot \nabla_{u} \lambda_{p}(\mathbf{u})$ for different points in phase space: (a) $u_{2}=\theta_{1}=\theta_{2}=0$ with different values of $u_{1}$, and (b) $u_{2}=0, \theta_{1}=4 \mathrm{~K}, \theta_{2}=2 \mathrm{~K}$ with different values of $u_{1}$. Since each characteristic field has both positive and negative values of $\mathbf{r}_{p} \cdot \nabla \lambda_{p}$, they are neither genuinely nonlinear nor linearly degenerate over all of phase space.

\subsection{Simple Waves that Do Not Break}

Here we illustrate simple wave solutions for the 2MSWE that do not break. For a simple wave $\mathbf{u}(x, t)=$ $\mathbf{U}(\sigma(x, t))$ to not break, it must satisfy $\mathbf{r}_{p}(\mathbf{U}) \cdot \nabla_{u} \lambda_{p}(\mathbf{U})=0$ for all values of $\sigma$. To find such a simple wave solution, one must first find a particular value of $\mathbf{U}_{\text {init }}$ and a characteristic field $p$ for which $\mathbf{r}_{p}\left(\mathbf{U}_{\text {init }}\right) \cdot \nabla_{u} \lambda_{p}\left(\mathbf{U}_{\text {init }}\right)=0$. Then, using $\mathbf{U}\left(\sigma_{\text {init }}\right)=\mathbf{U}_{\text {init }}$, one must solve (26) over an interval $\sigma_{-}<\sigma<\sigma_{+}$to see if $\mathbf{r}_{p}(\mathbf{U}) \cdot \nabla_{u} \lambda_{p}(\mathbf{U})=0$ over that interval. For the 2MSWE, the search for such solutions must be done numerically because the eigenstructure is analytically intractable. Points in phase space where $\mathbf{r}_{p} \cdot \nabla \lambda_{p}=0$ seem to occur infrequently based on the results in Figure 8 . In principle, one could search all of phase space to find all of these points, possibly using an iterative method. Here, instead, one family of non-breaking simple waves is presented as an example.

One family of non-breaking simple waves for the 2MSWE takes the form

$$
\mathbf{u}(x, t)=\mathbf{U}_{\text {init }}+\mathbf{r} \sigma(x-\lambda t),
$$

which is a special solution of (26)-(27) with constant eigenvector $\mathbf{r}_{p}(\mathbf{U}(\sigma))=\mathbf{r}$, constant eigenvalue $\lambda_{p}(\mathbf{U}(\sigma))=\lambda$, and traveling wave solution $\sigma(x, t)=\sigma(x-\lambda t)$. In this solution form, $\mathbf{U}_{\text {init }}$ acts as a background state and $\mathbf{r} \sigma(x-\lambda t)$ as an anomaly, but these are not just solutions to linearized perturbation equations; these are linearly degenerate solutions to the nonlinear $2 \mathrm{MSWE}$ (13). If $\mathbf{U}_{\text {init }}$ is chosen to be purely 2 nd baroclinic, then there is a simple wave of this form for each of the characteristic fields, as shown in Table 4 . The trivial choice $\mathbf{U}_{\text {init }}=0$ is a special case and was shown in Figure 4. Note that, for the purely mode- 2 values of $\mathbf{U}_{\text {init }}$ in Table 4 , the eigenvector $\mathbf{r}$ is either purely mode- 1 or purely mode- 2 . In summary, Table 4 demonstrates that the simple waves for all characteristic fields do not break if $\mathbf{U}_{\text {init }}$ is purely 2 nd baroclinic. On the other hand, if a 1st baroclinic contribution is included in $\mathbf{U}_{\text {init }}$, then a breaking simple wave might exist, which is the topic discussed next.

\subsection{Simple Waves that Break}

The results in Table 4 show that a nonzero mode- 1 background state is needed for a simple wave to break. One example of a breaking simple wave was given in the convergence test in Figure 2, where the background state was $\theta_{1}=4 \mathrm{~K}$ and $u_{1}=u_{2}=\theta_{2}=0$. As another example of a breaking simple wave, consider a background state with $u_{1}=5 \mathrm{~m} / \mathrm{s}, u_{2}=\theta_{1}=\theta_{2}=0$. The methods described in section 5.1 and appendix A were used to calculate "exact" simple wave solutions for this background state. The simple waves of all four characteristic fields will break with this background state. Figure 9 illustrates the eastward-propagating mode- 1 wave, which has a speed of roughly $50 \mathrm{~m} / \mathrm{s}$. Figure $9 \mathrm{a}$ shows the 
Table 4 Examples of simple waves that do not break. These are cases of $\mathbf{U}_{\text {init }}, \lambda$, and $\mathbf{r}$ for which (33) is a solution to the 2MSWE.

\begin{tabular}{|c|c|c|c|c|c|c|c|c|}
\hline \multicolumn{4}{|c|}{$\mathbf{U}_{i n i t}$} & \multirow{2}{*}{$\lambda(\mathrm{m} / \mathrm{s})$} & \\
\hline$u_{1}$ & $\theta_{1}$ & $u_{2}$ & $\theta_{2}$ & & $u_{1}$ & $\theta_{1}$ & $u_{2}$ & $\theta_{2}$ \\
\hline 0 & 0 & 0 & 0 & $\begin{array}{l}-50 \\
-25 \\
+25 \\
+50\end{array}$ & $\begin{array}{l}1 \\
0 \\
0 \\
1\end{array}$ & $\begin{array}{r}1 \\
0 \\
0 \\
-1\end{array}$ & $\begin{array}{l}0 \\
2 \\
2 \\
0\end{array}$ & $\begin{array}{r}0 \\
1 \\
-1 \\
0\end{array}$ \\
\hline 0 & 0 & $a$ & $b$ & $\begin{array}{r}50 \cdot\left(\frac{1}{\sqrt{2}} a-\sqrt{1+2 a^{2}-2 \sqrt{2} b}\right) \\
-25 \\
+25\end{array}$ & $\begin{array}{r}\frac{\lambda+\frac{1}{\sqrt{2}} a}{-1+2 \sqrt{2} b} \\
0 \\
0\end{array}$ & $\begin{array}{l}0 \\
0\end{array}$ & $\begin{array}{l}2 \\
2\end{array}$ & $\begin{array}{r}0 \\
1 \\
-1\end{array}$ \\
\hline & & & & $50 \cdot\left(\frac{1}{\sqrt{2}} a+\sqrt{1+2 a^{2}-2 \sqrt{2} b}\right)$ & $\frac{\lambda+\frac{1}{\sqrt{2}} a}{-1+2 \sqrt{2} b}$ & 1 & 0 & 0 \\
\hline
\end{tabular}

background shear, and Figures $9 \mathrm{~b}$ and c show snapshots at times $t=0$ and 4 hours, respectively, of pressure contours and the velocity field. The velocity field includes the background state and is shown in a reference frame moving eastward at $12 \mathrm{~m} / \mathrm{s}$. The plot at 4 hours was shifted in space to put the breaking wave in the center of the domain. Initially the wave was nearly sinusoidal, but after 4 hours, the gradients of both the pressure and velocity steepen sharply as the solution approaches the wave breaking time of $T_{*}=4.3$ hours. At $t=4$ hours, the wave resembles an internal bore (a.k.a. density current or gravity current) [24-27]. The flow is westward and nearly barotropic in the right half of the domain, and it sharply veers upward near $x=50 \mathrm{~km}$ where it meets the high pressure fluid in the lower left part of the domain. In the left half of the domain, the flow is mostly baroclinic. The simple waves for the other characterstic fields (not shown) have similar features but clearly several differences as well due to their different eigenstructures and propagation speeds.

For simple waves that break, the exact solution is no longer valid after characteristic lines meet. Defining weak solutions for nonconservative PDE is currently a challenging research topic that is beyond the scope of this paper (see [40-45] and references therein). Nevertheless, a numerical solution of a breaking wave will be shown to demonstrate that the numerical scheme proposed here can represent propagating discontinuities in a reasonable way.

The breaking wave in Figure 9 was also simulated numerically using the scheme from section 3. The domain is $100 \mathrm{~km}$ wide with periodic boundary conditions, and the grid spacing is $\Delta x=0.1 \mathrm{~km}$. The duration of the simulation was 36 hours, which is roughly 8 times the wave breaking time, and the results are shown in Figure 10. The time evolution of the energy is shown in Figure 10a, and the evolution of $u_{1}$ is shown in Figure 10b. The wave propagates eastward at roughly $50 \mathrm{~m} / \mathrm{s}$, but the snapshots shown in Figure 10b were shifted to put the discontinuity in the center of the domain. After the wave-breaking time $T_{*}=4.3$ hours, the energy of the wave anomaly decays to zero over a time scale of $\approx 10$ hours. Note that the total energy decays to a nonzero value corresponding to the energy of the background shear, $u_{1}=5 \mathrm{~m} / \mathrm{s}$. The energy here serves as a convex entropy function, and the breaking wave takes the form of a decaying $\mathrm{N}$-wave. This example demonstrates that the numerical scheme can represent propagating discontinuities without introducing spurious oscillations.

\subsection{Dam-Break Problem}

To illustrate a more complex situation with discontinuities, the well-known dam-break problem [38,39] is solved numerically with the scheme described in section 3 . Initially the flow is at rest $\left(u_{1}=u_{2}=0\right)$, and there is a jump in potential temperature at $x=0$ with cold air to the left:

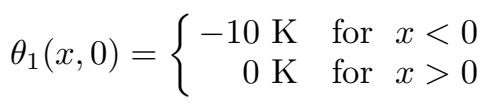

and $\theta_{2}=0$ over the whole domain initially. The grid spacing used is $\Delta x=0.5 \mathrm{~km}$.

Figure 11 shows the solution after 2 hours. The solution consists of four waves, with two propagating westward and two propagating eastward. Table 5 lists the propagation speed of each wave and the wave type based on the wavespeeds of the neighboring states. For instance, the leftmost front 


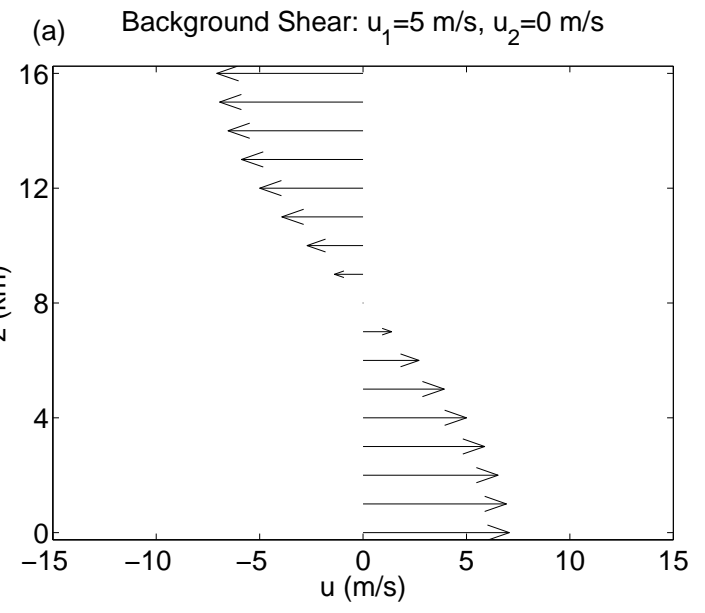

(b)

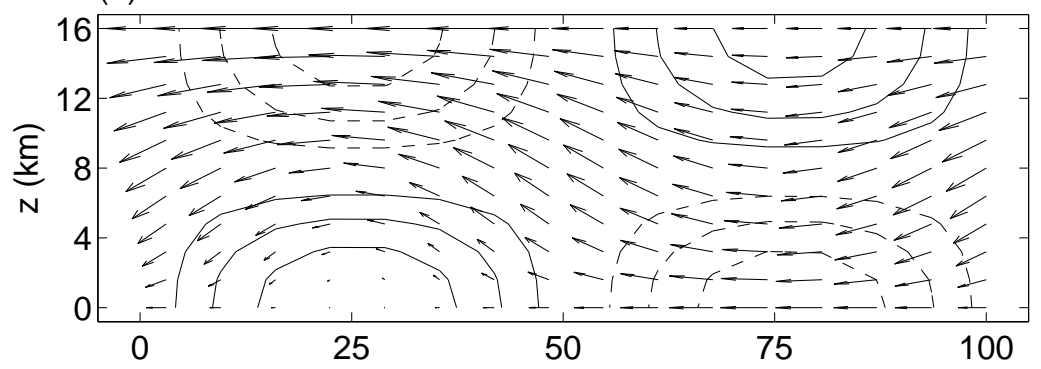

(c) $\quad \mathrm{t}=4$ hours

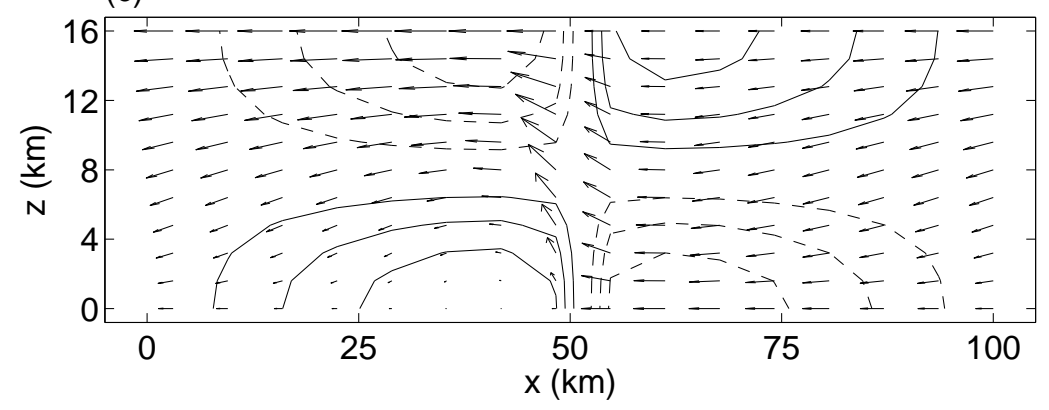

Fig. 9 Exact solution of a breaking simple wave in a background shear. The background shear uses $u_{1}=5$ $\mathrm{m} / \mathrm{s}$ and $u_{2}=0$ (a). Snapshots of the simple wave are shown at time $t=0$ hours (b) and $t=4$ hours (c). This is the mode- 1 simple wave that propagates eastward at roughly $50 \mathrm{~m} / \mathrm{s}$. The vector field includes the background shear and is plotted in a reference frame moving eastward at $12 \mathrm{~m} / \mathrm{s}$. The wave at $t=4$ hours has been shifted in space to put the breaking wave in the center of the domain. Pressure contours are shown with positive anomalies as solid lines and negative anomalies with dashed lines.

propagates into the cold region at $-57.6 \mathrm{~m} / \mathrm{s}$, which is intermediate between the wavespeeds of the states to its left and right $(-52.5$ and $-61.9 \mathrm{~m} / \mathrm{s}$, respectively). This is consistent with Lax's stability criterion for propagating fronts $[38,39,50,51]$. Therefore, this wave is identified as a genuinely nonlinear breaking wave. Also identified in Table 5 are a rarefaction fan and a linearly degenerate wave, while the fourth wave appears to be nearly linearly degenerate. This example shows that the numerical scheme can represent a variety of nonlinear waves: genuinely nonlinear waves, linearly degenerate waves, and rarefaction fans. 
(a)

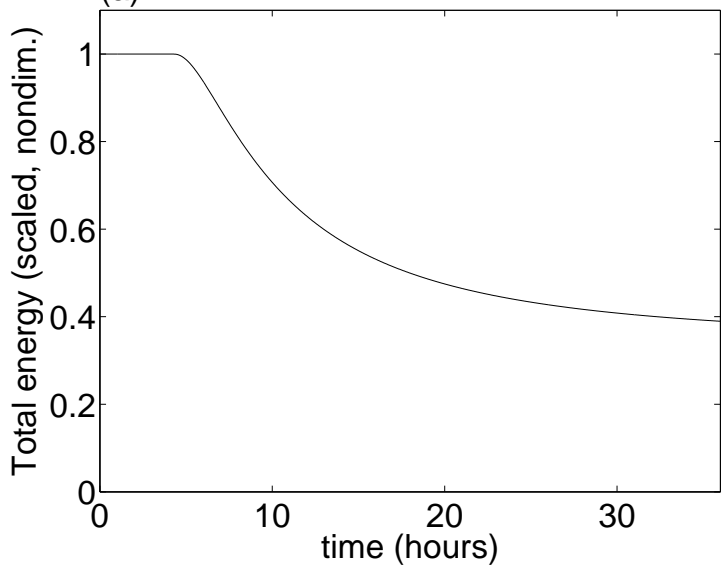

(b)

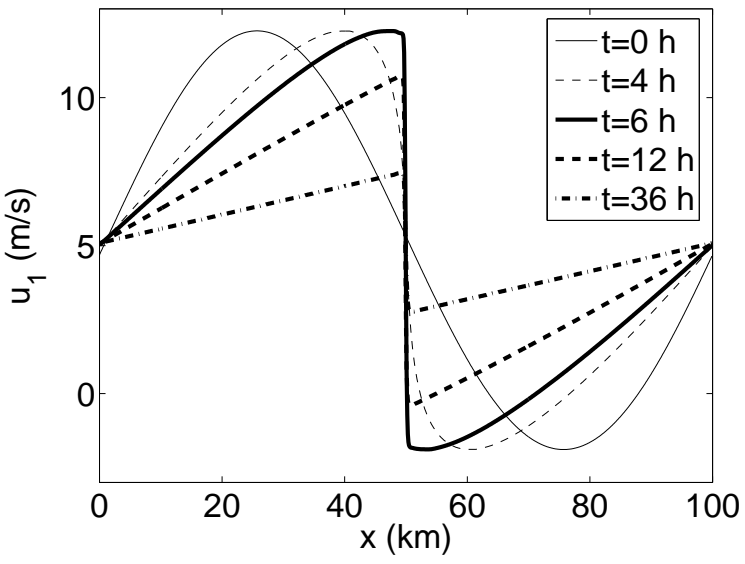

Fig. 10 Numerical solution of a breaking simple wave using the method proposed in section 3. (a) Time evolution of total energy, which decays to a nonzero value corresponding to the energy of the background shear, $u_{1}=5 \mathrm{~m} / \mathrm{s}$. (b) Snapshots of $u_{1}$ at times 0 (thin solid line), 4 (thin dashed line), 6 (thick solid line), 12 (thick dashed line), and 36 hours (thick dash-dot line). The wave breaks at time $T_{*}=4.3$ hours. The wave propagates eastward at roughly $50 \mathrm{~m} / \mathrm{s}$, but the snapshots were shifted to put the discontinuity in the center of the domain.
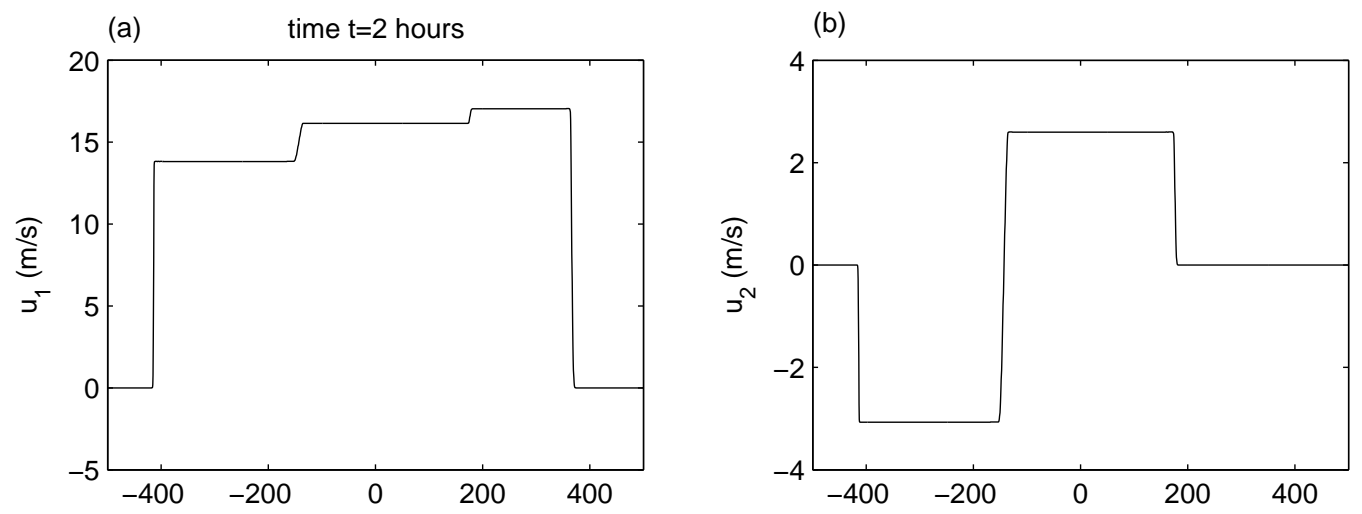

(c)
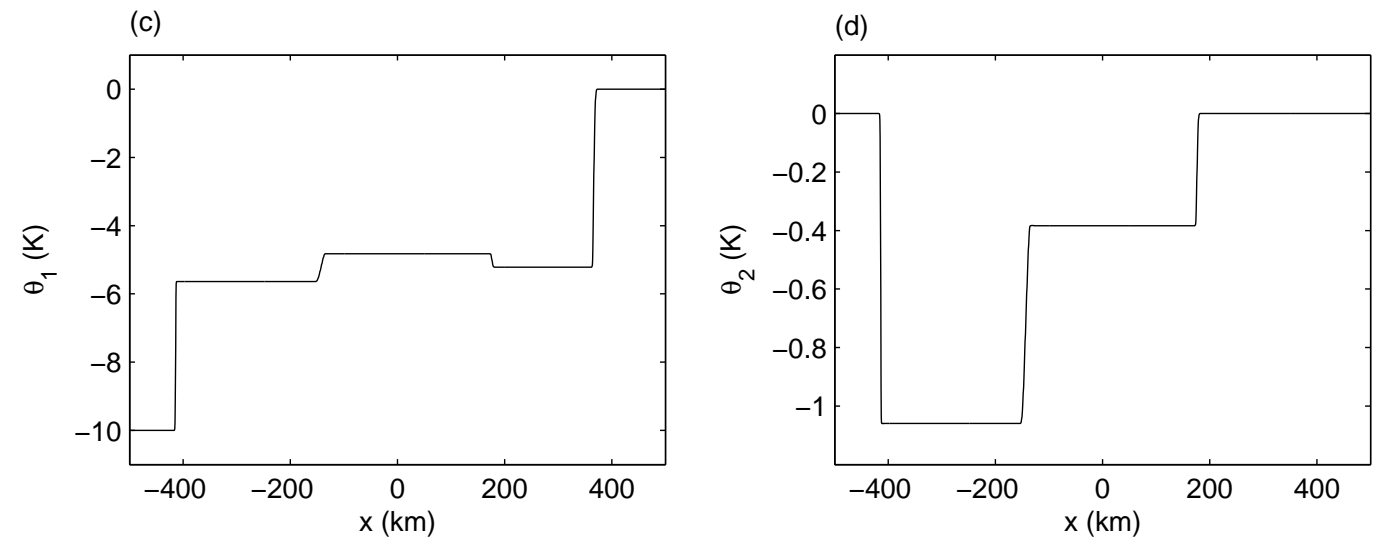

Fig. 11 Numerical solution of the dam-break problem. Snapshots are shown at time $t=2$ hours of (a) $u_{1}$, (b) $u_{2}$, (c) $\theta_{1}$, and (d) $\theta_{2}$. 
Table 5 Numerical solution of the dam-break problem. List of the four waves that emerge from the initial jump, along with the propagation speeds of the waves and the probable wave type. The probable wave type is inferred from the front speeds shown here and the wavespeeds of the neighboring states to the left and right of the front.

\begin{tabular}{l|l|l|l}
\hline $\begin{array}{l}\text { Front/fan location } \\
\text { at } t=2 \text { hours } \\
(\mathrm{km})\end{array}$ & $\begin{array}{l}\text { Propagation } \\
\text { speed (m/s) }\end{array}$ & $\begin{array}{l}\text { Relevant wavespeeds of } \\
\text { neighboring states to } \\
\text { left and right }(\mathrm{m} / \mathrm{s})\end{array}$ & Wave type \\
\hline-420 & -57.6 & -52.5 and -61.9 & Genuinely nonlinear \\
-150 & -20.5 to -19.3 & -20.6 and -19.1 & Rarefaction \\
170 & 24.6 & 24.6 and 24.4 & Nearly linearly degenerate \\
370 & 50.9 & 50.9 and 50.9 & Linearly degenerate \\
\hline
\end{tabular}

\section{Waves Generated by Thermal Forcing}

In this section, an important example from the tropical atmosphere is studied where gravity waves propagate through background wind shears. When convective clouds form and decay, condensational heating excites bore-like gravity waves that propagate away from the cloud. These waves play an important role in suppressing or promoting new convection in the vicinity of the pre-existent cloud. For simplicity, previous studies have examined these waves as they propagate through a motionless environment [10,52-55]. However, tropical convection often forms in environments with vertical shear of the horizontal winds $[16,28,29]$, and it is often not spatiotemporally isotropic but organized by waves on larger scales $[7,8,16]$. In order to understand how convection is organized on larger scales, it is important to study how the bore-like waves are affected by a background wind shear. This is the topic of this section.

To generate the bore-like waves, a localized heat source, is turned on at time $t=0$ and left on for the duration of the simulation, unlike the situation in (23). This is meant to represent heating from cloud formation $[10,52-54]$. The heat source takes the form

$$
S_{\theta 1}(x, t)=a \exp \left(-\frac{\left(x-x_{0}\right)^{2}}{2 \sigma^{2}}\right), \quad S_{\theta 2}(x, t)=-\frac{1}{4} S_{\theta 1},
$$

where the heating is centered in the middle of the domain at $x_{0}=1000 \mathrm{~km}$, the standard deviation is $\sigma=20 \mathrm{~km}$, and the amplitude is $a=200 \mathrm{~K} /$ day $\approx 8 \mathrm{~K} /$ hour. The vertical profile of this forcing is shown in Figure 12. It is a top-heavy heating that represents a combination of stratiform and deep convection (whereas the case in (23) was bottom-heavy, representing a combination of congestus and deep convection). The grid spacing is $\Delta x=2 \mathrm{~km}$ on a $2000 \mathrm{~km}$-wide domain.

Before considering the nonlinear case with a background shear, a motionless background state $u_{1}=u_{2}=\theta_{1}=\theta_{2}=0$ is considered for comparison. To further aide comparisons, the case with a motionless background state is shown for both linear dynamics (Figure 13) and nonlinear dynamics (Figure 14). Snapshots of velocity and potential temperature are shown at time $t=4$ hours. The mode- 1 heating forces a mode- 1 bore that travels at $50 \mathrm{~m} / \mathrm{s}$ and reaches $x=300$ and $1700 \mathrm{~km}$ at $t=4$ hours. At the front, the mode-1 bore has downward motion throughout the troposphere due to (8):

$$
w_{j}(x, t)=-\frac{1}{j} \partial_{x} u_{j}(x, t), \quad W(x, z, t)=w_{1}(x, t) \sqrt{2} \sin (z)+w_{2}(x, t) \sqrt{2} \sin (2 z) .
$$

Associated with this subsidence is adiabatic warming, which appears as the term $W d \theta_{b g} / d z$ in (1), and which is seen as the warming in $\theta_{1}$ by $1.2 \mathrm{~K}$ following the passage of the mode- 1 front. In a realistic situation with water vapor, this subsidence warming would cause a decrease in the convective available potential energy (CAPE) and stabilize the atmosphere for deep convection [3]. In this way, the mode-1 bore suppresses the formation of new convection. On the other hand, the mode- 2 bore has the opposite effect: it tends to promote convection. The mode- 2 heating forces a mode- 2 bore that travels at $25 \mathrm{~m} / \mathrm{s}$ behind the mode- 1 bore and reaches $x=650$ and $1350 \mathrm{~km}$ at $t=4$ hours. At the front there is upward motion at low levels, from which the low levels are cooled adiabatically. This is seen as the jump in $\theta_{2}$ of $-0.6 \mathrm{~K}$ as the mode- 2 bore passes. Thus the mode- 2 bore tends to promote new convection in the vicinity of the existing cloud by increasing the CAPE at low levels. These are the basic mechanisms 


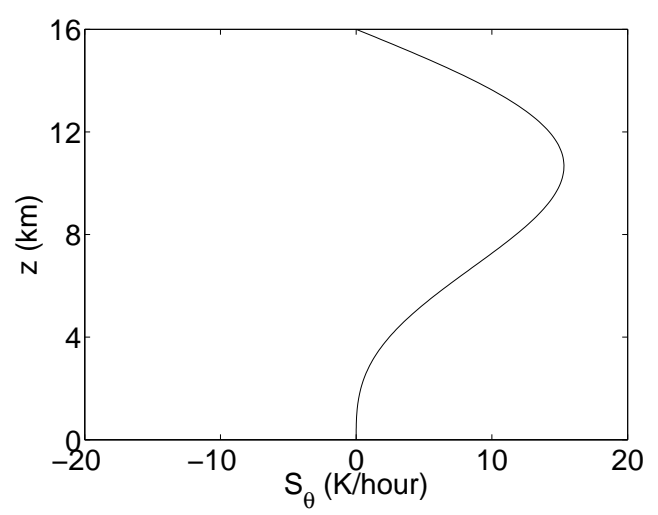

Fig. 12 Vertical profile of the heating (34) used to generate the waves shown in Figures 13-15.

of the mode- 1 and mode- 2 bores which are believed to play a central role in suppressing and favoring convection, respectively $[10,16,52-55]$.

When the nonlinear dynamics of the 2MSWE (13) are turned back on (but still with a motionless background state), the mode- 1 and mode- 2 responses are mixed, as shown in Figure 14 . The $25 \mathrm{~m} / \mathrm{s}$ bore is now reinforced by a mode- 1 response as well, but the $50 \mathrm{~m} / \mathrm{s}$ bore is almost purely 1st baroclinic. Although the mode- 1 and -2 reponses are now mixed, the effects are minor so that the previous discussion applies.

However, when both the nonlinearities and a non-zero background shear are included, the stucture of the traveling bores becomes spatially asymmetric: the waves to the east and west of the source are no longer the same. This is demonstrated in Figure 15, which was initialized with a background shear of $u_{1}=+10 \mathrm{~m} / \mathrm{s}$ and $u_{2}=-10 \mathrm{~m} / \mathrm{s}$, illustrated in Figure 15e. This shear profile was chosen to represent typical conditions in the westerly wind burst stage of the convectively active phase of the Madden-Julian oscillation, with strong westerlies at low- and mid-levels and easterlies at upper levels $[56,57]$. With this background shear, Figure 15 shows that the area to the west of the source is more favorable for convection than the area to the east of the source. The vertical profile of potential temperature is shown in Figure $15 \mathrm{f}$ for four different states at time $t=4$ hours. At $x=800 \mathrm{~km}$, after the westward-propagating mode- 2 bore passes, the atmosphere is in a state that is favorable for convection, with $\Theta<-1 \mathrm{~K}$ at low levels. To the east of the source, however, after the mode- 2 bore passes, the atmosphere is in a state that is less favorable for convection, with $\Theta \approx 0$ at low levels and $\Theta>3 \mathrm{~K}$ at upper levels. A detailed observational and computational study [57] of the westerly wind burst phase of the Madden-Julian oscillation has new organized convection always appearing westward of pre-existing organized convection, which agrees with the idealized study of the present paper. Thus, these effects might be important for the large-scale organization of convection.

\section{Conclusions}

A new set of PDE, the 2MSWE, were derived to capture the nonlinear interactions of gravity waves with different vertical profiles. The nonlinearities allow for waves interacting with a background wind shear, which is an important feature for many applications in the atmosphere. The nonlinear waves were shown to resemble internal bores, and the behavior of the waves was investigated for different background wind shears. When a background shear was included there was a pronounced asymmetry in the westward- and eastward-propagating waves. An idealized study of this asymmetry (Figure 15) in the westerly wind burst phase of the Madden-Julian oscillation produced a result in qualitative agreement with observations [57]; namely, new organized convection appears westward of existing organized convection in this jet shear. The westward-propagating waves produced an environment that is more favorable for convection than that produced by the eastward-propagating waves. This might be an important mechanism in the large-scale organization of tropical convection, since the convection is often not isotropic but organized on large scales by waves. 

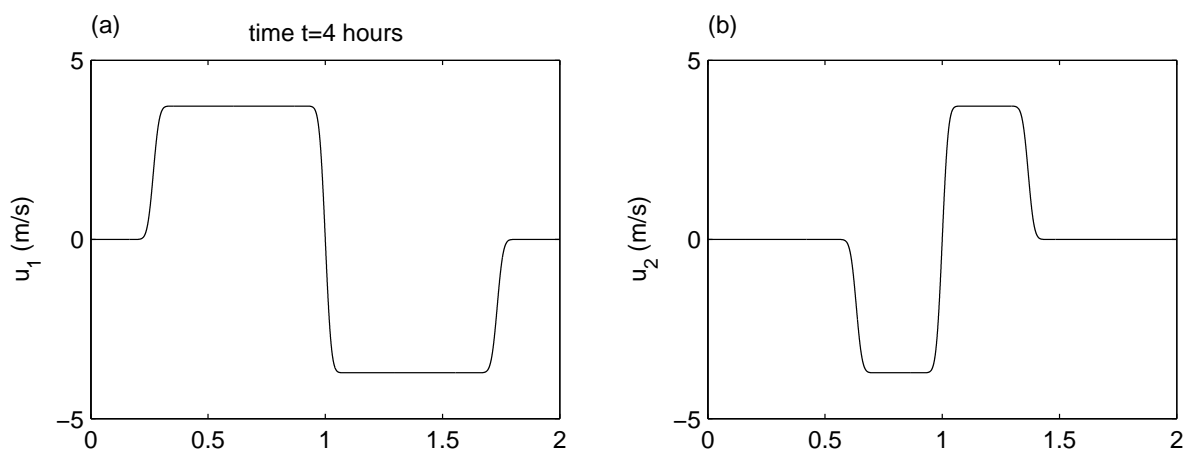

(c)
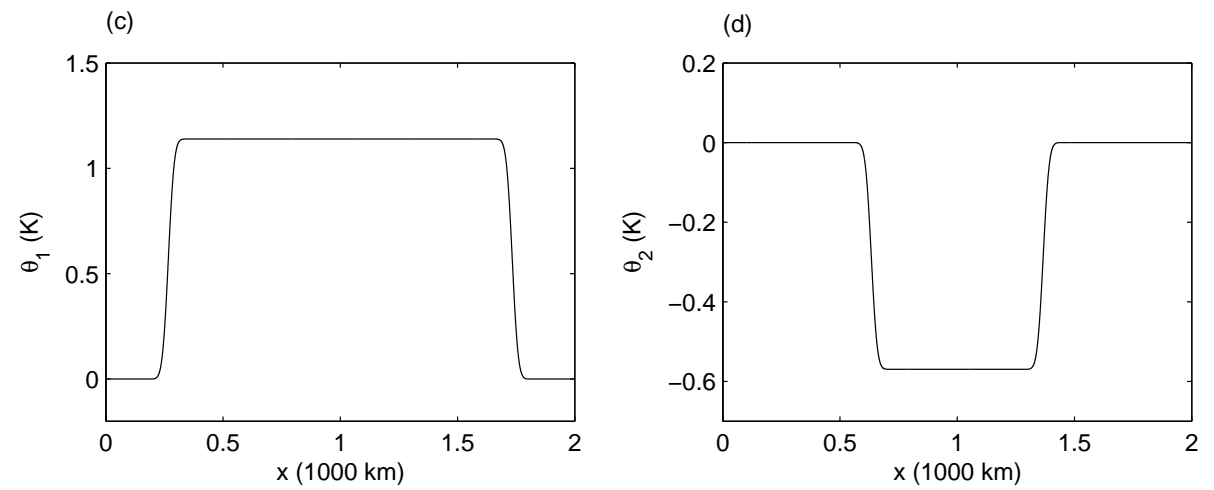

Fig. 13 Waves generated by the thermal forcing shown in (34). This case uses linear dynamics. The initial condition is motionless: $u_{1}=u_{2}=\theta_{1}=\theta_{2}=0$. Snapshots are shown at time $t=4$ hours for (a) $u_{1}$, (b) $u_{2}$, (c) $\theta_{1}$, and (d) $\theta_{2}$.
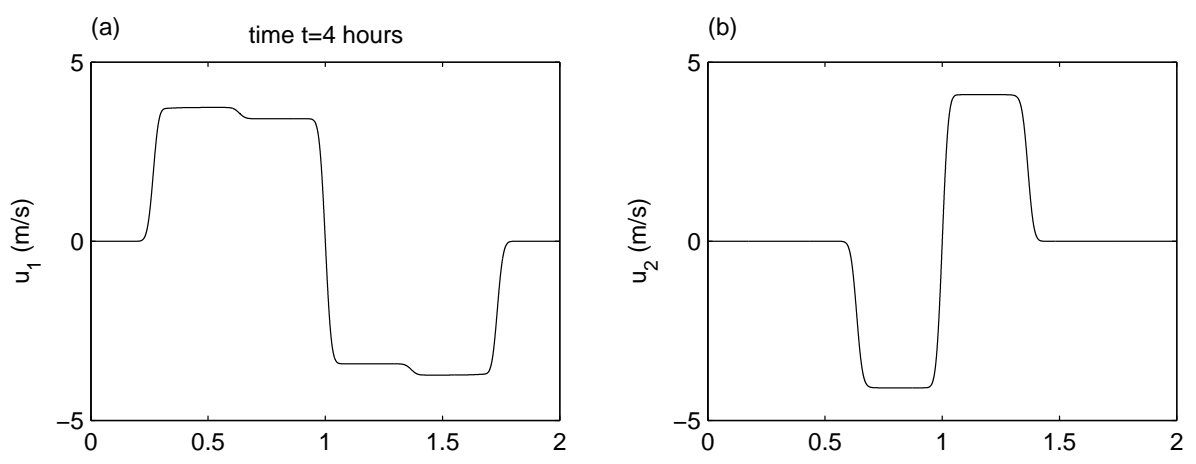

(c)

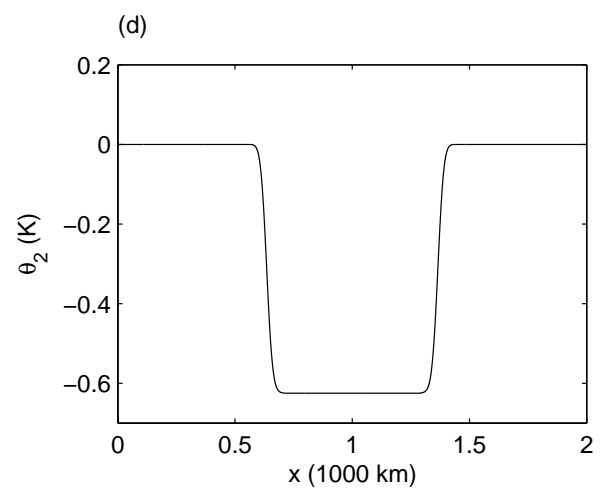

Fig. 14 Same as Figure 13 except the nonlinear dynamics of the 2MSWE (13) are used. 

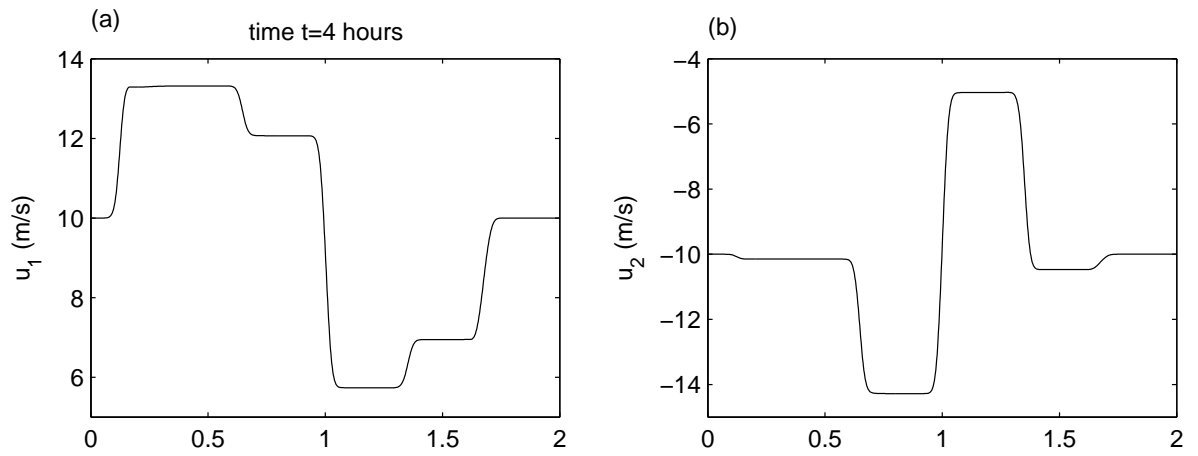

(c)

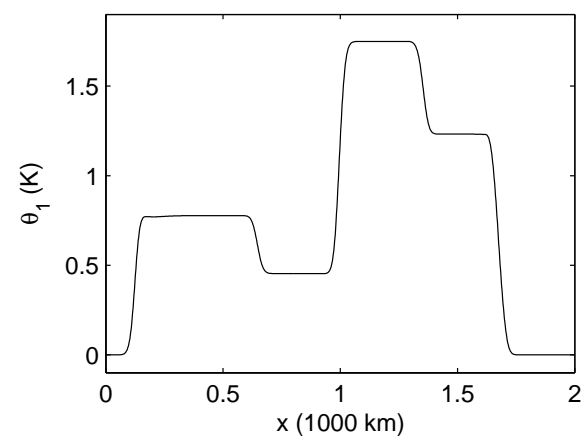

(d)

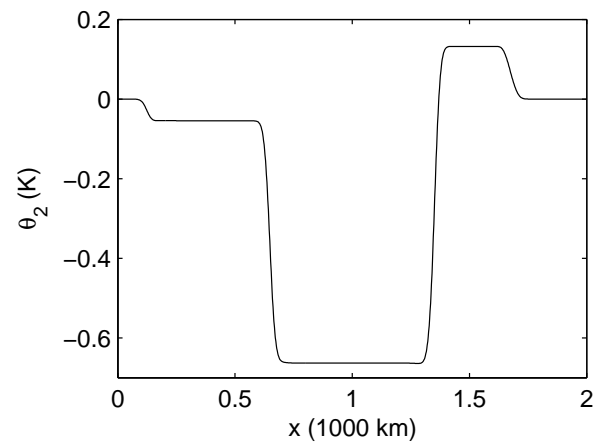

(e) Background Shear: $u_{1}=10 \mathrm{~m} / \mathrm{s}, u_{2}=-10 \mathrm{~m} / \mathrm{s}$
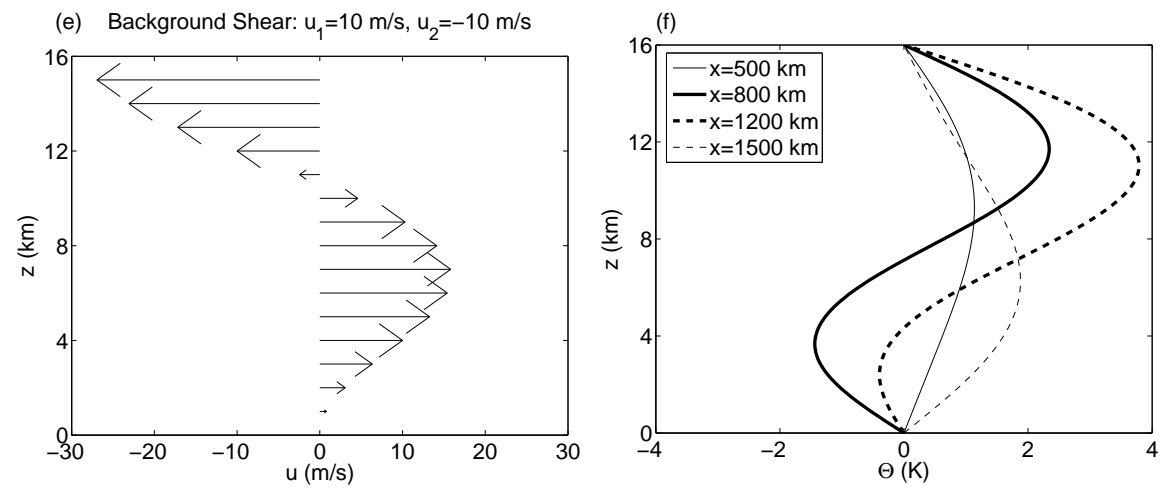

Fig. 15 Same as Figure 14 except a sheared initial condition is used: $u_{1}=10 \mathrm{~m} / \mathrm{s}, u_{2}=-10 \mathrm{~m} / \mathrm{s}, \theta_{1}=\theta_{2}=0$. (e) Vertical profile of the initial shear. (f) Vertical profiles of potential temperature at time $t=4$ hours at $x=500$ (thin solid line), $x=800$ (thick solid line), $x=1200$ (thick dashed line), and $x=1500 \mathrm{~km}$ (thick dashed line).

The 2MSWE were shown to have several interesting mathematical features: they are a system of nonconservative PDE with a conserved energy, they are conditionally hyperbolic, they are neither genuinely nonlinear nor linearly degenerate over all of phase space, and breaking waves can form from smooth initial conditions. Theory and numerics were developed to illustrate these features. When hyperbolicity is lost, the unstable waves have an overturning circulation that transports warm air upward to stabilize the system. Hyperbolic travelling waves were shown to be exact solutions to the nonlinear equations provided the background state is purely 2nd baroclinic. Such waves are linearly degenerate and do not break. In other cases, when a 1st baroclinic background state was present, several examples of breaking nonlinear waves were given. These are genuinely nonlinear waves that break, and they resemble internal bores (a.k.a. density currents or gravity currents) [24-27].

Due to these features of the 2MSWE, designing a numerical scheme for them is a challenge. A numerical method was presented in section 3 with simplicity and minimal computational cost as the main design principles. The non-conservative system is split into a conservative part and a non-conservative 
part. The conservative part is solved by a standard non-oscillatory central scheme [46,47], and the nonconservative part, which has a nilpotent advection matrix, is handled by a simple centered differencing scheme. The scheme was tested in sections 4 and 5 . When external source terms were applied to force the system into non-hyperbolic states, no catastrophic effects were introduced when hyperbolicity was lost, and the numerical solution did not seem to stray far from the hyperbolic region. When tested on breaking waves, the numerical scheme produced propagating discontinuties without intruducing spurious oscillations. A numerical solution to the dam-break problem demonstrated that the scheme can represent a variety of nonlinear waves: genuinely nonlinear waves, linearly degenerate waves, and rarefaction fans. These tests show that the numerical method can handle a variety of situations including non-hyperbolic states, nonlinear waves, and intense source terms.

Given the results shown here, the 2MSWE should be useful as a dynamical core for models that include the effects of water vapor and convection as interactive source terms [18-23]. For instance, this nonlinear dynamical core is important for the interactions of background wind shear with squall lines and mesoscale convective systems. The authors are currently pursuing this direction, and results will be presented elsewhere in the near future.

\section{A Appendix: Numerical Methods for Simple Waves}

In section 5, we introduced the simple wave solutions for the two-mode-shallow-water system in (13) and showed how such solutions are constructed by solving the ODE (26) and the scalar PDE (27). In this appendix it is shown how (26) and (27) are solved numerically to obtain the "exact" solutions shown in Figures 2 and 9.

First, the ODE (26) is solved. 20,000 discrete points are placed uniformly over the domain from $x=0$ to $100 \mathrm{~km}$, and $\sigma_{0}(x)$ is chosen to be a sinusoid with amplitude $\sigma_{a m p}$. This defines a set of discrete values of $\sigma$ in the interval $-\sigma_{a m p}<\sigma<\sigma_{a m p}$ with $\left.\mathbf{U}\right|_{\sigma=0}$ chosen to be the background state $\left(\theta_{1}=4 \mathrm{~K}\right.$ for Figure 2 and $u_{1}=5 \mathrm{~m} / \mathrm{s}$ for Figure 9$)$. A value of $\sigma_{a m p}=2 \mathrm{~m} / \mathrm{s}$ was used in Figure 2 and $\sigma_{a m p}=1 \mathrm{~m} / \mathrm{s}$ was used in Figure 9. The characteristic field $\left(\mathbf{r}_{p}, \lambda_{p}\right)$ chosen in each case is the one with a characteristic speed of roughly +50 $\mathrm{m} / \mathrm{s}$. The solution of the ODE (26) then gives the value of $\mathbf{U}$ for each of the 20,000 discrete values of $\sigma$, given its value at some initial point $\sigma_{j_{0}}$.

Second, the scalar PDE (27) is solved using characteristics as described in (28). This can be done to give an "exact" solution to the $2 \mathrm{MSWE}$ that is valid until the wave breaking time given by (30).

The wave breaking time given in (30) takes a simple form if two assumptions are made. First, the ODE (26) allows one to choose a normalization of $\mathbf{r}_{p}$. Here $\mathbf{r}_{p}$ is normalized by requiring

$$
\mathbf{r}_{p} \cdot \nabla \lambda_{p}=1
$$

assuming that $\mathbf{r}_{p} \cdot \nabla \lambda_{p}>0$ or $<0$ for all $\mathbf{U}$ in some region of phase space. With this normalization, it follows that

$$
\frac{d \lambda_{p}}{d \sigma}=\mathbf{r}_{p} \cdot \nabla \lambda_{p}=1
$$

so that

$$
\lambda_{p}=\lambda_{p}^{0}+\sigma, \quad \text { where } \quad \lambda_{p}^{0}=\left.\lambda_{p}\right|_{\sigma=0} .
$$

The PDE for $\sigma$ in (27) then takes the form of Burger's equation,

$$
\sigma_{t}+\left(\lambda_{p}^{0}+\sigma\right) \sigma_{x}=0,
$$

and the wave-breaking time $T_{*}$ also takes the simple form

$$
T_{*}=\frac{-1}{\min _{x} \frac{d \sigma_{0}}{d x}} .
$$

As the second simplifying assumption, as mentioned above, the initial conditions for $\sigma_{0}(x)$ are chosen to be sinusoidal,

$$
\sigma_{0}(x)=\sigma_{a m p} \sin (k x),
$$

so that the wave breaking time is simply

$$
T_{*}=\frac{1}{\sigma_{a m p} k} .
$$

For Figure 2 with $\sigma_{a m p}=2 \mathrm{~m} / \mathrm{s}$, the wave breaking time is $T_{*}=2.15$ hours, and for Figure 9 with $\sigma_{a m p}=1$ $\mathrm{m} / \mathrm{s}$, the wave breaking time is $T_{*}=4.3$ hours. 
Acknowledgements Samuel Stechmann is supported by a Department of Energy Computational Science Graduate Fellowship under grant DE-FG02-97ER25308. The research of Andrew Majda is partially supported by the National Science Foundation under grant NSF DMS-0456713 and the Office of Naval Research under grant ONR N00014-05-1-0164. Boualem Khouider is supported through a grant from the Natural Sciences and Engineering Research Council of Canada. 


\section{References}

1. Majda, A.J.: Introduction to PDEs and Waves for the Atmosphere and Ocean, Courant Lecture Notes in Mathematics, vol. 9. American Mathematical Society, Providence (2003)

2. Vallis, G.: Atmospheric and Oceanic Fluid Dynamics: Fundamentals and Large-scale Circulation. Cambridge University Press, New York (2006)

3. Emanuel, K.A.: Atmospheric Convection. Oxford University Press (1994)

4. Majda, A.J.: New multi-scale models and self-similarity in tropical convection. J. Atmos. Sci. 64, 1393-1404 (2007)

5. Baldwin, M., Gray, L., Dunkerton, T., Hamilton, K., Haynes, P., Randel, W., Holton, J., Alexander, M., Hirota, I., Horinouchi, T., Jones, D., Kinnersly, J., Marquardt C. andSato, K., Takahashi, M.: The quasibiennial oscillation. Rev. Geophys. 39(2), 179-229 (2001)

6. Zhang, C.: Madden-Julian Oscillation. Reviews of Geophysics 43, G2003+ (2005). DOI 10.1029/2004RG000158

7. Nakazawa, T.: Tropical super clusters within intraseasonal variations over the western Pacific. J. Met. Soc. Japan 66(6), 823-839 (1988)

8. Wheeler, M., Kiladis, G.N.: Convectively coupled equatorial waves: analysis of clouds and temperature in the wavenumber-frequency domain. J. Atmos. Sci. 56(3), 374-399 (1999)

9. Houze, R.A.: Mesoscale convective systems. Rev. Geophys. 42, G4003+ (2004). DOI 10.1029/2004RG000150

10. Mapes, B.: Gregarious tropical convection. J. Atmos. Sci. 50(13), 2026-2037 (1993)

11. Tulich, S., Mapes, B.: Multi-scale convective wave disturbances in the Tropics: Insights from a twodimensional cloud-resolving model. J. Atmos. Sci. p. in press (2007)

12. Tompkins, A.: Organization of tropical convection in low vertical wind shears: The role of cold pools. J. Atmos. Sci. 58(13), 1650-1672 (2001)

13. Gamache, J., Houze Jr, R.: Mesoscale air motions associated with a tropical squall line. Mon. Wea. Rev. 110(2), 118-135 (1982)

14. Mapes, B.E., Houze Jr., R.A.: Diabatic divergence profiles in western Pacific mesoscale convective systems. J. Atmos. Sci. 52, 1807-1828 (1995)

15. Haertel, P.T., Kiladis, G.N.: Dynamics of 2-day equatorial waves. J. Atmos. Sci. 61, 2707-2721 (2004)

16. Tulich, S.N., Randall, D., Mapes, B.: Vertical-mode and cloud decomposition of large-scale convectively coupled gravity waves in a two-dimensional cloud-resolving model. J. Atmos. Sci. 64, 1210-1229 (2007)

17. Mapes, B.E.: Convective inhibition, subgrid-scale triggering energy, and stratiform instability in a toy tropical wave model. J. Atmos. Sci. 57, 1515-1535 (2000)

18. Khouider, B., Majda, A.J.: A simple multicloud parameterization for convectively coupled tropical waves. Part I: Linear analysis. J. Atmos. Sci. 63, 1308-1323 (2006)

19. Khouider, B., Majda, A.J.: A simple multicloud parameterization for convectively coupled tropical waves. Part II: Nonlinear simulations. J. Atmos. Sci. 64, 381-400 (2007)

20. Khouider, B., Majda, A.J.: Model multicloud parameterizations for convectively coupled waves: Detailed nonlinear wave evolution. Dyn. Atmos. Oceans 42, 59-80 (2006)

21. Khouider, B., Majda, A.J.: Multicloud convective parameterizations with crude vertical structure. Theor. Comp. Fluid Dyn. 20, 351-375 (2006)

22. Khouider, B., Majda, A.J.: Multicloud models for organized tropical convection: enhanced congestus heating. J. Atmos. Sci. p. in press (2008)

23. Majda, A.J., Stechmann, S.N., Khouider, B.: Madden-Julian Oscillation analog and intraseasonal variability in a multicloud model above the equator. Proc. Natl. Acad. Sci. 104(24), 9919-9924 (2007)

24. Moncrieff, M., So, D.: A hydrodynamical theory of conservative bounded density currents. J. Fluid Mech. 198, 177-197 (1989)

25. Xu, Q., Moncrieff, M.: Density current circulations in shear flows. J. Atmos. Sci. 51(3), 434-446 (1994)

26. Klemp, J., Rotunno, R., Skamarock, W.: On the dynamics of gravity currents in a channel. J. Fluid Mech. 269, 169-198 (1994)

27. Klemp, J., Rotunno, R., Skamarock, W.: On the propagation of internal bores. J. Fluid Mech. 331, 81-106 (1997)

28. Grabowski, W.W., Wu, X., Moncrieff, M.W.: Cloud-resolving modeling of tropical cloud systems during Phase III of GATE. Part I: Two-dimensional experiments. J. Atmos. Sci. 53, 3684-3709 (1996)

29. LeMone, M., Zipser, E., Trier, S.: The role of environmental shear and thermodynamic conditions in determining the structure and evolution of mesoscale convective systems during TOGA COARE. Journal of the Atmospheric Sciences 55(23), 3493-3518 (1998)

30. Xue, M.: Density currents in shear flows: Effects of rigid lid and cold-pool internal circulation, and application to squall line dynamics. Quart. J. Roy. Meteor. Soc. 128, 47-73 (2002)

31. Majda, A.J., Biello, J.A.: The nonlinear interaction of barotropic and equatorial baroclinic Rossby waves. J. Atmos. Sci. 60, 1809-1821 (2003)

32. Khouider, B., Majda, A.J.: A non-oscillatory balanced scheme for an idealized tropical climate model: Part I: Algorithm and validation. Theor. Comp. Fluid Dyn. 19(5), 331-354 (2005)

33. Khouider, B., Majda, A.J.: A non-oscillatory balanced scheme for an idealized tropical climate model: Part II: Nonlinear coupling and moisture effects. Theor. Comp. Fluid Dyn. 19(5), 355-375 (2005)

34. Abgrall, R., Karni, S.: Two-layer shallow water systems: A relaxation approach. Submitted to SIAM J. Sci. Comput. (2007)

35. Ripa, P.: On improving a one-layer ocean model with thermodynamics. J. Fluid Mech. 303, 169-201 (1995) 
36. Schwendeman, D., Wahle, C., Kapila, A.: The Riemann problem and a high-resolution Godunov method for a model of compressible two-phase flow. J. Comp. Phys. 212(2), 490-526 (2006)

37. Deledicque, V., Papalexandris, M.: An exact Riemann solver for compressible two-phase flow models containing non-conservative products. J. Comp. Phys. 222(1), 217-245 (2007)

38. Evans, L.: Partial Differential Equations. American Mathematical Society (1998)

39. LeVeque, R.J.: Finite Volume Methods for Hyperbolic Problems. Cambridge University Press (2002)

40. Dal Maso, G., LeFloch, P., Murat, F.: Definition and weak stability of nonconservative products. J. Math. Pures Appl. 74(6), 483-548 (1995)

41. LeFloch, P.G., Tzavaras, A.E.: Representation of weak limits and definition of nonconservative products. SIAM Journal on Mathematical Analysis 30(6), 1309-1342 (1999). DOI 10.1137/S0036141098341794. URL http://link.aip.org/link/?SJM/30/1309/1

42. Crasta, G., LeFloch, P.: Existence result for a class of nonconservative and nonstrictly hyperbolic systems. Commun. Pure Appl. Anal. v1 pp. 1-18 (2002)

43. Bianchini, S.: On the Riemann problem for non-conservative hyperbolic systems. Archive for Rational Mechanics and Analysis 166(1), 1-26 (2003)

44. Toumi, I.: A weak formulation of Roe's approximate Riemann solver. J. Comp. Phys. 102(2), 360-373 (1992)

45. Pares, C.: Numerical methods for nonconservative hyperbolic systems: A theoretical framework. SIAM J. Numer. Anal. 44(1), 300-321 (2006)

46. Nessyahu, H., Tadmor, E.: Non-oscillatory central differencing for hyperbolic conservation laws. J. Comp. Phys. 87(2), 408-463 (1990)

47. Jiang, G.S., Tadmor, E.: Nonoscillatory central schemes for multidimensional hyperbolic conservation laws. SIAM J. Sci. Comput. 19(6), 1892-1917 (1998). DOI http://dx.doi.org/10.1137/S106482759631041X

48. Strang, G.: On the construction and comparison of difference schemes. SIAM J. Numer. Anal. 5, 506-517 (1968)

49. Charney, J.G., Eliassen, A.: On the growth of the hurricane depression. J. Atmos. Sci. 21, 68-75 (1964)

50. Majda, A.: Compressible Fluid Flow and Systems of Conservation Laws in Several Space Variables, Applied Mathematical Sciences, vol. 53. Springer-Verlag, New York (1984)

51. Lax, P.D.: Hyperbolic systems of conservation laws. II. Comm. Pure Appl. Math. 10, 537-566 (1957)

52. Bretherton, C.S., Smolarkiewicz, P.K.: Gravity waves, compensating subsidence and detrainment around cumulus clouds. J. Atmos. Sci. 46(6), 740-759 (1989)

53. Nicholls, M., Pielke, R., Cotton, W.: Thermally forced gravity waves in an atmosphere at rest. J. Atmos. Sci. 48(16), 1869-1884 (1991)

54. Pandya, R., Durran, D., Bretherton, C.: Comments on "thermally forced gravity waves in an atmosphere at rest". J. Atmos. Sci. 50(24), 4097-4101 (1993)

55. Lac, C., Lafore, J., Redelsperger, J.: Role of gravity waves in triggering deep convection during TOGA COARE. J. Atmos. Sci. 59(8), 1293-1316 (2002)

56. Lin, X., Johnson, R.H.: Kinematic and thermodynamic characteristics of the flow over the western Pacific warm pool during TOGA COARE. J. Atmos. Sci. 53, 695-715 (1996)

57. Wu, X., LeMone, M.: Fine structure of cloud patterns within the intraseasonal oscillation during toga coare. Monthly Weather Review 127(10), 2503-2513 (1999) 